\title{
Produits de matrices aléatoires et applications aux propriétés géometriques des sous-groupes du groupe linéaire
}

\author{
YVES GUIVARC'H \\ Université de Paris VI, Laboratoire de Probabilités, Tour 56-3e étage, 4, \\ Place Jussieu, 75252 Paris Cedex 05, France
}

(Received 25 April 1988 and revised 15 December 1989)

Abstract. Using the asymptotic properties of products of random matrices we study some properties of the subgroups of the linear group. These properties are centered around the theorem of $\mathrm{J}$. Tits giving the existence of free subgroups in linear groups.

\section{Introduction}

L'objet de ce travail est de montrer comment des méthodes de la théorie des systèmes dynamiques et du calcul des probabilités permettent d'obtenir des propriétés algébriques ou géometriques des sous-groupes du groupe linéaire. En fait, dans le cas d'un groupe linéaire sur un corps local, l'introduction d'une mesure de probabilité $\mu$, de support contenu dans un tel sous-groupe $\Gamma$ et engendrant un semi-groupe fermé $T_{\mu}$, permet au moyen du produit de matrices aléatoires associé, et en particulier de ses exposants de Liapunoff de traduire de manière quantitative des propriétés qualitatives de $\Gamma$ ou $T_{\mu}$. Par exemple on donne dans la partie 1 une démonstration du théorème de $\mathrm{J}$. Tits concernant l'existence de sous-groupes libres non abéliens dans un groupe linéaire [23], démonstration dont l'argument essentiel repose sur le théorème ergodique multiplicatif d'Osedelets au lieu de la densité au sens de Zariski. Utilisant les méthodes développées on obtient par une application simple du théorème de super-rigidité que toute représentation $(d>2)$ d'un réseau de $\mathrm{Sl}(d, \mathbb{R})$ dans un espace de dimension inférieure a une image finie. Afin de poursuivre l'étude des propriétés sous-jacentes au théorème de Tits on introduit dans la partie 2 , pour un sous-groupe $\Gamma$ de $S I(d, \mathbb{R})$, l'ensemble des points limites dans la compactification de Moore de l'espace symmétrique correspondant. On donne en particulier des résultats concernant l'action de $\Gamma$ sur l'ensemble $L_{\Gamma}$. de ces points limites et on montre que $L_{1}$. est de dimension de Hausdorff positive. On précise cette propriété par une étude de la mesure $\mu$-invariante portée par $L_{1}$. Cette étude conduit au retour à des propriétés nouvelles des produits de matrices aléatoires qui éclairent le théorème de Tits: sous une condition d'irréductibilité et de proximalité de l'action de $T_{\mu}$ sur l'espace projectif, un tel produit a presque sûrement une valeur propre dominante simple, réelle, et son comportement asymptotique est donné par le plus grand exposant de Liapunoff. Ceci répond à une conjecture de J. Cohen et 
H. Fürstenberg [3]. Sous une hypothèse renforcée on peut en déduire des résultats plus précis concernant le spectre d'un tel produit qui est alors asymptotiquement simple et réel. Observons d'autre part qu'une autre direction naturelle d'application de ces techniques concerne l'étude de certaines algèbres de groupe intervenant en théorie des représentations unitaires [15]. Dans des divers résultats un rôle essentiel est joué par l'étude du spectre de Liapunoff d'un produit de matrices aléatoires indépendantes et en particulier de sa simplicité, étude qui a été faite en [10] et précisée en [7], [12]. Les techniques utilisées ici sont donc susceptibles de compléter ou de se combiner à celles de géométrie algébrique traditionnellement utilisées dans ce contexte comme cela apparaît également en [5], [18], [26].

\section{Sous-groupes libres du groupe lineaire}

\subsection{Introduction}

Dans un travail fondamental [23], J. Tits a donné une démonstration du théorème suivant.

THÉORÈme 1. Soit $k$ un corps de caractéristique nulle, $\Gamma$ un sous-groupe du groupe linéaire $\mathrm{Gl}(d, k)$. Alors, ou bien $\Gamma$ contient un sous-groupe résoluble d'indice fini, ou bien $\Gamma$ contient un groupe libre à deux générateurs.

La preuve de $\mathbf{J}$. Tits est basée sur les propriétés de la topologie de Zariski et des considérations de géométrie algébrique. L'objet de cette partie 1 est d'en donner une autre démonstration, suivant le même plan, mais utilisant plutôt les propriétés des produits de matrices aléatoires. Cette méthode permet plusieurs simplifications et est susceptible d'applications diverses. Très brièvement résumé, le plan de la preuve de J. Tits est d'abord de remplacer $k$ par un corps localement compact convenable $K$ tel que, sur un certain espace projectif $P^{r}(K), \Gamma$ agisse de manière 'suffisamment irréductible et contractante'; puis la proposition cruciale 3-11 permet de trouver dans $\Gamma$ 'suffisament' d'éléments $g$ tels que $g$ et $g^{-1}$ aient des valeurs propres dominantes simples, enfin la densité au sens de Zariski permet de trouver dans $\Gamma$ deux éléments 'en position générale', et un argument classique montre qu'ils engendrent un sous-groupe libre. On suivra ici cette démarche mais la technique sera différente et en particulier les propriétés des produits de matrices aléatoires permettront de suppléer à la proposition 3-11 de [23].

\subsection{Produits de matrices aléatoires}

Rappelons d'abord le théorème ergodique multiplicatif d'Oseledets sur un corps $K$ commutatif localement compact non discret (corps local) [21], dont la valeur absolue sera notée |.|.

ThÉORÈme D'OSELEDETS. Soit $(\Omega, \theta, \pi)$ un système dynamique ergodique, $g(\omega)$ une fonction de $\Omega$ à valeurs dans $G l(d, K)$ telle que $\log \|g(\omega)\|$ et $\log \left\|g^{-1}(\omega)\right\|$ soient $\pi$-integrables. Supposons $g_{n}(\omega)=g \circ \theta^{n}(\omega)$ et $S_{n}(\omega)(n \in Z)$ défini par

$$
\begin{aligned}
S_{n}(\omega) & =g_{n-1} g_{n-2} \cdots g_{0} \quad(n \geq 0) \\
S_{0}(\omega) & =\text { Id } \\
S_{-n}(\omega) & =g_{-n}^{-1} g_{-n+1}^{-1} \cdots g_{-1}^{-1} \quad(-n<0) .
\end{aligned}
$$


Alors il existe une matrice diagonale par blocs et une fonction $\varphi(\omega)$ à valeurs matricielles telle que

$$
S_{n}(\omega)=\varphi \circ \theta^{n}(\omega) \Delta_{n} \varphi^{-1}(\omega) .
$$

De plus pour tout $x$ correspondant à chaque bloc on $a$ :

$$
\lim _{n \rightarrow+\infty}(1 / n) \log \left\|S_{n}(\omega) \varphi(\omega) x\right\|=\lambda^{i}
$$

(où $\lambda^{i}$ est compté avec la multiplicité du bloc correspondant).

Remarque. Les nombres $\lambda^{i}$ sont bien définis et s'appellent exposants caractéristiques. Ils seront ordonnés par $\lambda^{1} \geq \lambda^{2} \geq \cdots \geq \lambda^{d}$.

Définition 1. On dira qu'un semi-groupe $T$ opère de manière proximale sur un espace métrique $(E, \delta)$ si pour tous $x, y$ de $E$ on peut trouver une suite $t_{n} \in T$ avec $\lim _{n} \delta\left(t_{n} x, t_{n} y\right)=0$.

On considèrera la situation $T \subset G l(d, K)$ opérant sur l'espace projectif $P^{d-1}(K)$ par les transformations projectives naturelles où $K$ sera dans la suite un corps local. Définition 2. On dira que le semi-groupe $T \subset G l(d, K)$ opère de manière totalement irréductible sur $P^{d-1}(K)$ s'il n'existe pas de réunion finie de sous-espaces projectifs laissée invariante par $T$.

Soint maintenant $\mu$ une mesure de probabilité portée par le sous-groupe $\Gamma$ de $G l(d, K)$ et $T_{\mu}$ le semi-groupe engendré par le support $\sum_{\mu}$ de $\mu$. On note $\Omega$ l'espace produit $\left(\Sigma_{\mu}\right)^{Z}, \pi$ la probabilité canonique produit $\mu^{Z}$ sur $\Omega, \theta$ le décalage et $g_{n}(\omega)$ les fonctions coordonnées qui sont ici des variables aléatoires indépendantes de loi $\mu$. La condition d'intégrabilité de $g_{0}(\omega)$ sera toujours supposée satisfaite. On a établi en [10] le théorème suivant si $K=\mathbb{R}$, et sa démonstration s'étend au cas d'un corps local $[8]$.

ThÉORÈME 2. Soit $\mu$ une mesure de probabilité sur $G l(d, \mathbb{R})$ et supposons que $T_{\mu}$ opère de manière proximale et totalement irréductible sur $P^{d-1}(\mathbb{R})$. Alors les deux premiers exposants caractéristiques du produit des matrices aléatoires $g_{n}(\omega)$ sont différents. De plus la loi de la direction dilatante $\varphi(\omega) e_{1}$ correspondant au plus grand exposant ne charge pas de sous-espace projectif.

Remarque. Si $T_{\mu}$ est un groupe, les propriétés de proximalité et d'irréductibilité envisagées dans le théorème restent valables sur l'espace projectif dual formé des hyperplans de $P^{d-1}(K)$. Il en découle, puisque $\Delta_{n}$ est alors changée en $\left(\Delta_{n}^{\prime}\right)^{-1}$ que les deux derniers exposants du produit initial sont distincts également sous l'hypothèse du théorème [cf. 12].

Définition 3. On dira que $g \in G l(d, K)$ admet une valeur propre dominante simple s'il existe $a^{+} \in P^{d-1}(K)$ et $H^{-}$hyperplan projectif supplémentaire avec:

$$
\forall x \notin H^{-} \lim _{n \rightarrow+\infty} g^{n} x=a^{+} .
$$

On dira que $a^{+}$est le point attractif de $g$ et $H^{-}$son hyperplan répulsif. La condition de proximalité du théorème est alors assurée par la. 
Proposition 1. Soit $\Gamma$ un groupe opérant de manière totalement irréductible sur $P^{d-1}(K)$ et supposons qu'il existe un élément de $\Gamma$ ayant une valeur propre dominante simple. Alors $\Gamma$ opère de manière proximale sur $P^{d-1}(K)$.

Preuve. Pour $x$ et $y$ dans $P^{d-1}(K)$, il suffit de trouver $\eta \in \Gamma$ avec $\eta x \notin H^{-}, \eta y \notin H^{-}$ où $H^{-}$est le sous-espace répulsif de $\gamma \in \Gamma$ et $a^{+}$son point attractif. Alors en effet:

$$
\lim _{n} \gamma^{n} \eta x=\lim _{n} \gamma^{n} \eta y=a^{+} \text {. }
$$

Or la condition $\eta x \in H^{-}$ou $\eta y \in H^{-}$pour tout $\eta$ de $\Gamma$ s'écrirait $x \in \eta^{-1}\left(H^{-}\right)$ou $y \in \eta^{-1}\left(H^{-}\right)$et violerait donc la totale irréductibilité dans le dual de $P^{d-1}(K)$, c'est-á-dire dans $P^{d-1}(K)$ d'après la remarque précédente.

Remarque. Cette proposition et la remarque précédente conduisent à une certaine symétrie dans les résultats: les deux premiers exposents et les deux derniers sont simultanément différents si $T_{\mu}=\Gamma$.

\subsection{Itération d'une matrice et produits de matrices aléatoires}

Ici la dimension $d$ de l'espace vectoriel $K^{d}$ sera 2 au moins.

Définition 4. On appellera doublet de points-hyperplans (ou simplement doublet) un système de deux points $a^{+}, a^{-}$de $P^{d-1}(K)(d \geq 2)$ et de deux hyperplans $H^{+}$, $H^{-}$, avec les relations $a^{+} \notin H^{-}, a^{-} \notin H^{+}, a^{+} \in H^{+}, a^{-} \in H^{-}$et on le notera $D\left(a^{+}, a^{-}, H^{+}, H^{-}\right)$.

Si $g \in G l(d, K)$ est tel que $g$ et $\left(g^{\prime}\right)^{-1}$ admettent des valeurs propres dominantes simples, les points attractifs $a^{+}, a^{-}$et les sous-espaces répulsifs $H^{+}, H^{-}$correspondants forment un doublet que l'on notera $D(g)$.

De même le théorème précédent et la remarque qui suit montre que si $T_{\mu}=\Gamma$ est un sous-groupe de $G l(d, K)$ totalement irréductible et proximal sur $P^{d-1}(K)$, les sous-espaces dilatants et contractants des cocycles $S_{n}(\omega),\left[S_{n}(\omega)^{\prime}\right]^{-1}$ donnent lieu à un doublet point hyperplan que l'on notera $D(\omega)$ et qui est formé de $a^{+}=\varphi(\omega) e_{1}$, $a^{-}=\varphi(\omega) e_{d}, H^{-}=\varphi(\omega) e_{1}^{\prime}, H^{+}=\varphi(\omega) e_{d}^{\prime}$ où $e_{1}^{\prime}$ et $e_{d}^{\prime}$ désignant les hyperplans d'annulation de la première et de la dernière coordonnée.

Définition 5. On dira que deux doublets $D\left(a^{+}, a^{-}, H^{+}, H^{-}\right)$et $D\left(b^{+}, b^{-}, L^{+}, L^{-}\right)$ sont en position générale si l'on a $b^{+} \notin H^{+} \cup H^{-}, b^{-} \notin H^{+} \cup H^{-}, a^{+} \notin L^{+} \cup L^{-}$, $a^{-} \notin L^{+} \cup L^{-}$.

Il est clair que les couples de doublets en position générale forment un ouvert dense.

Proposition 2. Soit $\Gamma \subset G l(d, K)$ un sous-groupe totalement irréductible et proximal sur $P^{d-1}(K), \mu$ une mesure de probabilité, portée par $\Gamma$ et telle que $T_{\mu}=\Gamma$. Alors le produit de matrices aléatoires $S_{n}(\omega)$ définit pp un doublet $D(\omega)$. De plus pour presque tout $\left(\omega, \omega^{\prime}\right) \in \Omega \times \Omega$, les doublets correspondants $D(\omega)$ et $D\left(\omega^{\prime}\right)$ sont en position générale.

Preuve. L'existence de $D(\omega)$ découle du Théorème 2 et de la remarque qui le suit. Fixons $D(\omega)$ et montrons la négligeabilité de l'ensemble des $\omega^{\prime}$ tels que $D(\omega)$ et $D\left(\omega^{\prime}\right)$ ne soient pas en position générale. Sinon il existerait $\Omega^{\prime} \subset \Omega$ de mesure 
positive vérifiant par exemple une condition $\varphi\left(\omega^{\prime}\right) e_{1} \in \varphi(\omega)\left(e_{1}^{\prime} \cup e_{d}^{\prime}\right)$ pour $\omega^{\prime} \in \Omega^{\prime}$; la loi de $\varphi\left(\omega^{\prime}\right) e_{1}$ (qui est la direction dilatante) chargerait une réunion de deux hyperplans projectifs, ce qui est impossible d'aprés le Théorème 2.

Proposition 3. Dans la situation du théorème d'Oseledets, supposons le premier et le dernier exposants de multiplicité 1 et soil $D(\omega)$ le doublet associé au cocycle $S_{n}(\omega)$. Alors pour presque tout $\omega$ il existe une sous-suite $n_{k}(\omega)$ telle que $S_{n_{k}}(\omega)$ et $S_{n_{k}}^{-1}(\omega)$ aient des valeurs propres dominantes simples. De plus on a $\lim _{k \rightarrow \infty} D\left[S_{n_{k}}(\omega)\right]=D(\omega)$.

La preuve découlera de deux lemmes dont le premier résulte du théorème des fonctions implicites et est bien connu.

LEMME 1. Soit $K$ un corps local $u$ un endomorphisme linéaire de $K^{d}$ ayant une valeur propre dominante simple, $a^{+}$le point attractif et $\mathrm{H}^{-}$le sous-espace répulsif associés. Soit $u_{n} \in E$ nd $\left(K^{d}\right)$ avec $\lim u_{n}=u$. Alors, pour $n$ assez grand, $u_{n}$ a une valeur propre dominante simple, un point attractif $a^{+}$, un sous-espace répulsif $H_{n}^{-}$vérifiant:

$$
\lim _{n} a_{n}^{+}=a^{+}, \lim _{n} H_{n}^{-}=H^{-} \text {. }
$$

LEMME 2. Soit $(\Omega, \Theta, \pi)$ un système dynamique ergodique, $\varphi$ une application mesurable de $\Omega$ dans $G I(d, K)$. Alors pour presque tout $\omega$, il existe une sous-suite $n_{k}(\omega)$ avec

$$
\lim _{k \rightarrow \infty} \varphi^{-1}(\omega) \varphi \circ \theta^{n_{k}}(\omega)=\text { Id. }
$$

Preuve. Il est commode de munir $G l(d, K)$ d'une distance invariante à gauche $\delta$. Si l'assertion du lemme est fausse, il existe $\Omega^{\prime} \subset \Omega$ de mesure positive et $\varepsilon>0$ avec

$$
\lim \inf \delta\left[I, \varphi^{-1}(\omega) \varphi \circ \theta^{n}(\omega)\right]=\varepsilon>0 .
$$

Puisque les boules $B_{\delta}(g,(\varepsilon / 2))$ recouvrent $G l(d, K)$ si $g$ parcourt $G$, on peut trouver $\gamma \in G l(d, K)$ tel que l'ensemble $\Omega^{\prime \prime} \operatorname{des} \omega^{\prime} \in \Omega^{\prime}$ avec $\delta\left[\gamma, \varphi\left(\omega^{\prime}\right)\right]<(\varepsilon / 2)$ soit de mesure positive.

Le théorème de récurrence de Poincaré donne pour presque tout $\omega^{\prime} \in \Omega^{\prime \prime}$ une sous-suite $n_{k}$ avec $\theta^{n_{k}} \omega^{\prime} \in \Omega^{\prime \prime}$. Alors, pour $\omega^{\prime} \in \Omega^{\prime \prime}$.

$$
\delta\left[\varphi\left(\omega^{\prime}\right), \varphi \circ \theta^{n_{k}}\left(\omega^{\prime}\right)\right] \leq \delta\left[\varphi\left(\omega^{\prime}\right), \gamma\right]+\delta\left[(\gamma), \varphi \circ \theta^{n_{k}}\left(\omega^{\prime}\right)\right]<\varepsilon
$$

ce qui contredit $\lim _{n}$ inf $\delta\left[\varphi\left(\omega^{\prime}\right), \varphi \circ \theta^{n_{h}}\left(\omega^{\prime}\right)\right] \geq \varepsilon$.

Preuve de la proposition. Ecrivons, d'après le théorème d'Oseledets:

$$
\begin{aligned}
S_{n}(\omega) & =\varphi \circ \theta^{n}(\omega) \Delta_{n} \varphi^{-1}(\omega) \\
S_{n}^{-1}(\omega) & =\varphi(\omega) \Delta_{n}^{-1} \varphi^{-1} \circ \theta^{n}(\omega)
\end{aligned}
$$

et montrons d'abord l'existence des valeurs propres dominantes. Il revient au même de considérer

$$
\begin{aligned}
\varphi^{-1}(\omega) S_{n}(\omega) \varphi(\omega) & =v_{n}(\omega) \Delta_{n} \\
\varphi^{-1}(\omega) S_{n}^{-1}(\omega) \varphi(\omega) & =\Delta_{n}^{-1} v_{n}^{-1}(\omega) \quad \text { avec } \quad v_{n}(\omega)=\varphi^{-1}(\omega) \varphi \circ \theta^{n}(\omega)
\end{aligned}
$$

Notons que d'après le Théorème 2 et la remarque on a

$$
\Delta_{n} e_{1}=\lambda_{n}^{-1} e_{1}, \quad \Delta_{n} e_{d}=\lambda_{n}^{d} e_{d} \text { avec } \Delta_{n}^{\prime}=\Delta_{n} / \lambda_{n}^{1}, \Delta_{n}^{\prime \prime}=\lambda_{n}^{d} \Delta_{n}^{-1}
$$

convergeant vers les projecteurs canoniques $\Delta^{\prime}$ et $\Delta^{\prime \prime}$ sur $K e_{1}$ et $K e_{d}$. On est donc 
amené à considérer $v_{n}(\omega) \Delta_{n}^{\prime}$ et $\Delta_{n}^{\prime \prime} v_{n}^{-1}(\omega)$. Or, par exemple:

$$
\left\|v_{n}(\omega) \Delta_{n}^{\prime}-\Delta^{\prime}\right\| \leq\left\|v_{n}(\omega)-I\right\|\left\|\Delta_{n}^{\prime}\right\|+\left\|\Delta_{n}^{\prime}-\Delta^{\prime}\right\|
$$

et

$$
\lim _{k \rightarrow+\infty}\left\|v_{n_{k}}(\omega)-I\right\|=0
$$

d'après le Lemme 2. Comme $\lim _{h \rightarrow \infty}\left\|\Delta_{n}^{\prime}-\Delta^{\prime}\right\|=0$, on a bien $\lim _{k \rightarrow \infty} \| v_{n_{k}}\left(\omega^{\prime}\right) \Delta_{n}^{\prime}-$ $\Delta^{\prime} \|=0$ et le Lemme 1 permet de conclure. On traite de même $\left\|\Delta_{n}^{\prime \prime} v_{n}(\omega)-\Delta^{\prime \prime}\right\|$ avec la même sous-suite $n_{k}$. Au niveau des doublets point-hyperplan associés on a

$$
D\left[S_{n}(\omega)\right]=\varphi(\omega) D\left[v_{n}(\omega) \Delta_{n}^{\prime}\right]=\varphi(\omega) D\left[\Delta_{n}^{\prime \prime} v_{n}(\omega)\right]
$$

et la convergence de $D\left[S_{n_{k}}(\omega)\right]$ vers $D(\omega)$ résulte encore de celle de $v_{n_{k}}(\omega)$ vers $I$ et du Lemme 1. Les Propositions 2 et 3 donnent alors le.

ThÉORÈME 3. Soit $K$ un corps local, $\Gamma \subset G l(d, K)$ un sous-groupe totalement irréductible et proximal sur $P^{d-1}(K),(d \geq 2), \mu$ une mesure de probabilité portée par $\Gamma$ et telle que $T_{\mu}=\Gamma$. Notons $S_{n}(\omega)=g_{n} g_{n-1} \cdots g_{0}$ le produit des matrices alèatoires indépendantes $g_{i}$ de loi $\mu$. Alors, pour presque tout $\omega$, il existe une sous-suite $n_{k}(\omega)$ telle que $S_{n_{k}}(\omega)$ et $S_{n_{k}}^{-1}(\omega)$ aient des valeurs propres dominantes simples. De plus, pour presque tous $\omega$ et $\omega^{\prime}$ de $\left(\Sigma_{\mu}\right)^{Z}$, il existe des sous-suites $n_{k}(\omega)$ et $n_{p}^{\prime}\left(\omega^{\prime}\right)$ telles que $S_{n_{k}}(\omega)$ et $S_{n^{\prime} \rho}\left(\omega^{\prime}\right)$ engendrent un groupe libre non abélien.

Preuve. La première partie découle des premières parties des Proposition 2 et 3 . Utilisant les secondes parties de ces propositions on trouve, pour presque tout $\left(\omega, \omega^{\prime}\right) \in \Omega \times \Omega$ des sous-suites $n_{k}(\omega), n_{p}^{\prime}\left(\omega^{\prime}\right)$ et des doublets $D(\omega), D\left(\omega^{\prime}\right)$ en position générale avec:

$$
\begin{aligned}
& \lim _{k \rightarrow+\infty} D\left[S_{n_{k}}(\omega)\right]=D(\omega) \\
& \lim _{k \rightarrow+\infty} D\left[S_{n_{p}^{\prime}}\left(\omega^{\prime}\right)\right]=D\left(\omega^{\prime}\right) .
\end{aligned}
$$

Pour $n_{k}$ et $n_{p}^{\prime}$ assez grands, $D\left[S_{n_{k}}(\omega)\right]$ et $D\left[S_{n_{p}^{\prime}}\left(\omega^{\prime}\right)\right]$ sont donc aussi en position générale. La conclusion découle alors du lemme suivant [23] dont nous reproduisons la preuve.

LeMme 3. Soient $g$ et $h$ deux éléments de $G l(d, K)$ tels que $g, h, g^{-1}, h^{-1}$ aient des valeurs propres dominantes simples et supposons $D(g), D(h)$ en position générale. Alors il existe un entier $n$ tel que $g^{n}$ et $h^{n}$ engendrent un groupe libre.

Preuve. Notons $D(g)=D\left(a^{+}, a^{-}, H^{+}, H^{-}\right), D(h)=D\left(b^{+}, b^{-}, L^{+}, L^{-}\right)$et observons que

$$
\begin{aligned}
& \lim _{n \rightarrow \pm x} g^{n}\left(b^{+} \cup b^{-}\right)=a^{ \pm} \\
& \lim _{n \rightarrow \pm x} h^{n}\left(b^{+} \cup b^{-}\right)=b^{ \pm} .
\end{aligned}
$$

Ceci donne deux voisinages $A$ et $B$ de $a^{+} \cup a^{-}$et $b^{+} \cup b^{-}$et $n \in N$ tels que:

$$
g^{m}(B) \subset A, \quad h^{m}(A) \subset B \quad(|m| \geq n) .
$$

Soient alors $p \notin A \cup B$ et $u=g^{n}, v=h^{n} \cdot g^{m} p \in A, h^{m} p \in B(|m| \geq h)$. 
Si $\lambda(u, v)$ est un mot quelconque en $u$ et $v$ on a $\lambda(u, v) p \in A \cup B$.

Si $\lambda$ est non trivial et donc $\lambda(u, v) p \neq p, \lambda(u, v) \neq \mathrm{Id}$.

Le groupe engendré par $u$ et $v$ est donc libre.

\subsection{Groupes non résolubles et proximalité}

Afin de démontrer le Théorème 1, il est commode de considérer des groupes finiment engendrés et, pour abréger le langage de donner la définition.

Définition. On dit qu'un groupe $G$ est presque résoluble (resp. nilpotent) s'il possède un sous-groupe d'indice fini résoluble (resp. nilpotent).

On est amené à manipuler diverses représentations d'un groupe, par extension du corps de base notamment. Rappelons qu'une représentation de $G$ sur un corps $k$ est absolument irréductible si elle reste irréductible par toute extension du corps de base. Il suffit d'une extension de degré fini du corps de base pour obtenir, à partir d'une représentation donnée, une nouvelle représentation dont les quotients de la suite de Jordan-Hölder sont absolument irréductibles.

L'objet de ce paragraphe est de montrer le.

THÉORÈme 4. Soit $V$ un espace vectoriel de dimension finie sur un corps $k$ de caractéristique zéro, $G \subset G I(V)$ un sous-groupe finiment engendré et non presque résoluble. Alors il existe un corps local $K$, un homomorphisme de $k$ dans $K$, une représentation $\rho$ de dimension 2 au moins d'un sous-groupe d'indice fini $G^{\prime}$ de $G$ sur le corps $K$ telle que $\rho\left(G^{\prime}\right)$ soit totalement irréductible et qu'il existe $g \in G^{\prime}$ tel que $\rho(g)$ ait une valeur propre dominante simple.

Remarques. On emploiera ici l'expression $\rho(G)$ totalement irréductible au lieu de $\rho(G)$ opère de manière totalement irréductible sur l'espace projectif associé.

On peut toujours remplacer $k$ par l'extension de degré fini de $Q$ engendrée par les coefficients des matrices representant un nombre fini de générateurs de $G$.

La preuve va découler de modifications successives de la représentation initialement donnée de $G$ dans $G l(V)$.

Définition. On dira qu'une représentation de $G$ dans un espace vectoriel $V$ sur un corps $k$ est totalement absolument irréductible s'il n'existe pas d'extension du corps de base $k$, de sous-groupe d'indice fini $H$ de $G$, telle que, dans la nouvelle représentation $H$ laisse invariant un sous-espace strict.

Lem fini du corps de base, la représentation de $G$ dans $G l(V)$ totalement absolument irréductible.

Preuve. On considère une suite de Jordan-Hölder de la représentation donnée, à quotients successifs $V_{i+1} / V_{i}(0 \leq i \leq r)$. Le noyau de la représentation naturelle de $G$ dans $\oplus_{i=0}^{r} V_{i+1} / V_{i}$ est nilpotent et donc dans l'un au moins des $G l\left(V_{i+1} / V_{i}\right)$ l'image de $G$ n'est pas presque résoluble, et la représentation de $G$ correspondante peut être supposée absolument irréductible par extension finie du corps de base. Fixons alors cette représentation et notons encore $V_{i+1} / V_{i}$ par $V$. Considérons alors les triplets $\left(H, k^{\prime}, W\right)$ formés d'un sous-groupe distingué d'indice fini $H$ de $G$, d'une 
extension $k^{\prime}$ de degré fini de $k$ et d'un sous-espace $W$ de $V \otimes k^{\prime}$ laissé invariant par $H$. Fixons un tel sous-espace $W$ de dimension minimum, le corps $k^{\prime}$ et le sous-groupe $H$ correspondants. Par construction, la représentation de $H$ dans $W$ est totalement absolument irréductible et $G / H$ s'identifie à un groupe de permutation fini puisque la représentation de $G$ dans $V$ est fidèle et absolument irréductible. Il en découle que $H$, comme $G$, n'est pas presque résoluble et en particulier $\operatorname{dim} W \geq 2$. L'énoncé du lemme s'obtient donc en remplaçant $G$, par $H$ et $V$ par $W$.

Lемме 5. Soit $V$ un espace vectoriel de dimension finie sur un corps $k$ de caractéristique zéro, $G \subset G l(V)$ un sous-groupe finiment engèndré du groupe linéaire de $V$. On suppose que, dans une clôture algébrique de $k$, les quotients des valeurs propres de tout $g$ de $G$ soient racines de l'unité. Alors $G$ est presque nilpotent.

Preuve. On peut considérer la représentation naturelle de $G$ dans End $V: u \rightarrow g u g^{-1}$ dont le noyau est central dans $G$ et on peut donc supposer les valeurs propres de tout $g$ de $G$ racines de l'unité. On considère alors une suite de Jordan-Hölder de $V$ et l'on note $\rho_{l}$ les représentations irréductibles dans les quotients successifs. Le noyau de la représentation somme directes des $\rho_{i}$ est nilpotent et d'après un théorème de Schur [24] relatif aux groupes linéaires périodiques tous les $\rho_{i}(G)$ sont finis. Il en découle que $G$ possède un sous-groupe nilpotent d'indice fini.

Preuve du théorème. Soit $G \subset G l(V)$ totalement absolument irréductible et, d'après le Lemme 5, $a \in G$ tel que les quotients des valeurs propres de a dans une clôture algébrique de $k$ ne soient pas racines de l'unité. Par extension de degré fini de $Q$, si $\lambda_{i} / \lambda_{j}$ n'est pas racine de l'unité, on peut trouver un corps local $(K,|\cdot|)$, un homomorphisme $\sigma$ de $k$ dans $K$ tel que $\left|\sigma\left(\lambda_{i} / \lambda_{j}\right)\right| \neq 1$ ([23], [25]). Pour abréger les notations on identifiera dans la suite $k$ à $\sigma(k) \subset K$.

Donc $\left|\lambda_{i}\right| \neq\left|\lambda_{j}\right|$ et il y a une valeur propre $\lambda_{i}$ dont le module $\left|\lambda_{1}\right|$ est maximum et réalisé avec multiplicité $p<\operatorname{dim} V$. Désignons par $e_{i}(1 \leq i \leq p)$ une base de la somme des sous-espaces caractéristiques correspondants, base dans laquelle la matrice de a est triangulaire. Alors dans $\Lambda^{p}(V \otimes K)$, le vecteur $e=e_{1} \wedge \cdots \wedge e_{p}$ sera vecteur propre de $\Lambda^{p} a$ et la valeur propre correspondante $\rho$ dominera toutes les autres en module. On va maintenant remplacer $\Lambda^{p}(V \otimes K)$ par un sous-espace contenant $e$. Puisque la représentation de $G$ dans $\Lambda^{p}(V \otimes K)$ est complètement réductible [2], $e$ appartiendra à l'une des composantes irréductibles de $\Lambda^{p}(V \otimes K)$ et l'on notera $\eta$ la représentation dans $W \subset \Lambda^{p}(V \otimes K)$ ainsi obtenue. Soit enfin $W_{1}$ un sous-espace de dimension minimum fixé par un sous-groupe d'indice fini et distingué $L$ de $G$, $\Lambda$ un système de représentants de $G / L$ dans $G$ : on a $W=\sum_{\gamma \epsilon \wedge} \gamma W_{1}$, chacun des $\gamma W_{1}$ est fixé par $L$ et les représentations de $L$ dans chaque $\gamma W_{1}$ sont totalement irréductibles. Montrons que $e$ appartient à l'un des $\gamma W_{1}$. Pour tout $w \in W$ on $a$ :

$$
\begin{array}{ll}
\text { soit } & \lim _{n \rightarrow+\infty}\left(\eta\left(a^{n}\right) w\right) /\left(\rho^{n}\right)=0 \\
\text { soit } & \lim _{n \rightarrow+\infty}\left[\left(\eta\left(a^{n}\right) w /\left(\rho^{n}\right)\right] \in K^{*} e\right.
\end{array}
$$

et l'ensemble des $w$ satisfaisant la première relation est un hyperplan $E$. D'autre part $a^{n}$ appartient à $L$ le long d'une progression arithmétique et laisse donc alors 
invariant $\bigcup_{\gamma \in \wedge} \gamma W_{1}$; comme ce dernier ensemble engendre $W$, il n'est pas contenu dans l'hyperplan $E$ et $e$ appartient bien à $\bigcup_{\gamma \in \wedge} \gamma W_{1}$, donc à l'un des $\gamma W_{1}$. On a donc remplacé $G$ par $L, W$ par $\gamma W_{1}$ et il reste à voir que $\operatorname{dim} \gamma W_{1} \geq 2$. Sinon $L$ fixerait la droite engendrée par $e$ et donc le sous-espace engendré par $e_{1}, e_{2}, \ldots, e_{p}$ dans $V \otimes K$. Ceci contredit le fait que $G$ soit totalement absolument irréductible dans $V$. La représentation $\rho$ de l'énoncé est donc la restriction de $\Lambda^{p} \eta$ à $\gamma W_{1}$, le sous-groupe $G^{\prime}$ est égal à $L$ et l'élément $g$ est une puissance convenable de $\Lambda^{p}$ a qui admet clairement une valeur propre dominante simple.

\subsection{Preuve du Théorème 1}

Ce théorème découle du Théorème 1 ' et d'un lemme.

THÉORÈME 1'. Soit $k$ un corps de caractéristique nulle, $\Gamma$ un groupe finiment engendré de $G l(d, k)$. Alors ou bien $\Gamma$ contient un groupe résoluble d'indice fini, ou bien $\Gamma$ contient un groupe libre à 2 générateurs.

Preuve. Supposons la première alternative non satisfaite et appliquons le Théorème 4 du paragraphe précédent: on peut remplacer $k$ par un corps local $K$, et $\Gamma$ par un sous-groups d'indice fini opérant de manière proximale et totalement irréductible sur $P^{d-1}(K)(d \geq 2)$. D'après le Théorème $3 \mathrm{du} \S 1.3, \Gamma$ contient alors un groupe libre à deux générateurs. Les techniques élémentaires de géométrie algébrique [2] donnent une démonstration simple du lemme:

Lемме. Soit $\Gamma$ un sous-groupe de $G l(d, k)$ tel que tout sous-groupe finiment engendré soit presque résoluble. Alors $\Gamma$ est presque résoluble.

Preuve. On utilisera encore le théorème de structure des groupes périodiques linéaires dû è Schur [22]: un tel groupe contient un groupe abélien d'indice fini.

Soit $G_{0} \subset \bar{\Gamma}$ la composante connexe en topologie de Zariski de l'élément neutre dans l'adhérence algébrique $\bar{\Gamma}$ de $G$. Soit $\Gamma_{i}(i \in I)$ famille croissante de sous-groupes de $\Gamma$ finiment engendrés de réunion égale à $\Gamma, \Gamma_{i}$ leurs adhérences algébriques et $G_{i}$ les composantes neutres correspondantes. Puisque $G_{i}$ est d'indice fini dans $\bar{\Gamma}_{i}$, les $G_{i}$ sont résolubles connexes et forment une famille croissante. Si $R$ est un élément de dimension maximum, $\Gamma_{i} / \Gamma_{i} \cap R$ est donc fini et $\Gamma / \Gamma \cap R$ est réunion croissante de sous-groupes finis. Il en est de même de son image dans la représentation naturelle de $\Gamma$ dans $\mathscr{G}_{0} / \mathscr{R}$ où $\mathscr{G}_{0}$ et $\mathscr{R}$ désignent les algèbres de Lie de $G_{0}$ et $R$ : le théorème de Schur déjà cité implique que cette image possède un sous-groupe abélien d'indice fini. On peut d'autre part supposer $\Gamma \subset G_{0}$ car $G_{0}$ est d'indice fini dans $\bar{\Gamma}$ et alors le noyau de la représentation considérée commute avec $G_{0}$ modulo $R: \Gamma / \Gamma \cap R$ contient un groupe résoluble d'indice fini et il en est de même de $\Gamma$.

Ce lemme montre que pour démontrer le Théorème 1 , on peut toujours se placer dans la situation du Théorème $1^{\prime}$.

\subsection{Application aux représentations des réseaux}

On prouve ici, en application des techniques précédentes le.

THÉORÈme 5. Soit $\Gamma$ un réseau de $\mathrm{Sl}(d, \mathbb{R}),(d>2), \rho$ une représentation de $\Gamma$ dans un espace vectoriel $k^{n}$ où $n<d$ et $k$ est un corps de caractéristique 0 . Alors $\rho(\Gamma)$ est fini. 
Preuve. Si $n=1, \rho(\Gamma)$ est commutatif et la propriété de Kajdan pour $\Gamma$ [26] implique $\rho(\Gamma)$ fini.

Dans le cas général on va appliquer le Théorème 4 ou plutôt les arguments de sa preuve, avec $G=\rho(\Gamma)$, l'espace de $\rho$ étant $V$ avec $\operatorname{dim} V<d$ : d'après la propriété de Kajdan, $\rho(\Gamma)$ n'est pas presque résoluble s'il est infini. On peut de plus supposer d'après le Lemme $4, \rho(\Gamma)$ totalement absolument irréductible. Comme $\Gamma$ est de type fini et $\rho(\Gamma)$ non presque résoluble, on peut remplacer comme au début de la preuve du Théorème 4 , le corps de base $k$ par un corps local $K$ de façon que pour un certain $a \in \rho(\Gamma) \subset \mathrm{Gl}(n, K)$, il y ait une valeur propre dominante dont le module est répété $p$ fois au plus ( $p<n$ ). Montrons d'abord que, du fait que $n<d$, l'adhérence (topologique) $\overline{\rho(\Gamma)}$ dans $G l(n, K)$ est moyennable. Considérons pour cela l'adhérence algébrique de $\rho(\Gamma)$ et une composante $H$ presque simple et connexe de sa partie semi-simple: on a ainsi un homomorphisme $\pi$ du réseau $\Gamma$ dans $H$ dont l'image est algébriquement dense et on va voir que $\pi(\Gamma)$ est relativement compacte an appliquant le théorème de super-rigidité de G. A. Margulis, et en distinguant donc suivant la nature de $K[18]$.

Si $K=\mathbb{R}$, on peut supposer que $H$ est $R$-simple en passant au quotient par le centre (fini) et si l'ensemble des points réels $H_{\mathbb{B}}$ était non compact, $\pi$ se prolongerait en un isomorphism de $\mathrm{S} 1(d, \mathbb{R})$ sur $H_{\mathbb{R}}$, ce qui est exclu car $n<d, \operatorname{dim} H_{\mathbb{R}}<d$. Donc $H_{\mathbb{R}}$ est compact et de même que $\overline{\pi(\Gamma)}$.

Si $K=\mathbb{C}$, on peut encore supposer $H$ simple et l'isomorphisme entre $\mathrm{Sl}(d, \mathbb{C})$ et $H$ est encore exclu, ce qui donne $\overline{\pi(\Gamma)}$ compact.

Si $K$. est ultramétrique, $\overline{\pi(\Gamma)}$ est compact, Finalement $\rho(\Gamma)$ est contenu dans une extension d'un groupe résoluble par un groupe compact et $\overline{\rho(\Gamma)}$ est donc moyennable. On considère alors, comme dans la preuve du Théorème 4 , la sous-représentation totalement irréductible dans $W_{1} \subset \Lambda^{P} V$ qui y est construite et qui contient le vecteur propre dominant de $\Lambda^{p} a$. Puisque $\overline{\rho(\Gamma)}$ est moyennable, son action sur l'espace projectif associé à $W_{1}$ laisse une mesure de probabilité invariante; à cause des propriétés de $\Lambda^{p} a$ et de la totale irréductibilité de l'action de $\rho(\Gamma)$, cette mesure est concentrée au point attractif de $\Lambda^{p} a$, ce qui contredit l'irréductibilité de $W_{1}$ et $\operatorname{dim} W_{1} \geq 2$. D'où $\rho(\Gamma)$ est presque résoluble, donc fini d'après la propriété de Kajdan.

\section{Sur les points limites de certains sous-groupes du groupe lineaire}

\subsection{Introduction}

Si $\Gamma$ est un sous-groupe discret de $S I(2, \mathbb{R})$; l'action de $\Gamma$ sur le disque unité $D$ par transformations conformes permet de définir la notion de points limites qui joue un rôle essentiel dans l'étude du flot géodésique sur la surface $D / \Gamma$. L'ensemble $L_{\mathrm{l}}$. de ces points limites soit égal au cercle unité, soit du type de Cantor, si $\Gamma$ est non dégénéré. De plus l'action de $\Gamma$ sur $L_{\boldsymbol{l}}$ possède des propriétés particulières, la minimalité par exemple. Dans cette partie on donne une extension à $S l(d, \mathbb{R})$ de la notion de points limites d'un sous-groupe fermé $\Gamma \subset S l(d, \mathbb{R})$. On montre en particulier que si $\Gamma$ est 'non dégénéré', la dimension de Hausdorff de $L_{1}$ est strictement 
positive, en utilisant les propriétés des produits de matrices aléatoires ([5], [10], [12]). On étudie l'action de $\Gamma$ sur $L_{1}$ et certaines mesures naturelles sur $L_{\Gamma}$. Ceci permet, en retour, de préciser les propriétés du spectre d'un tel produit.

\subsection{Rappel sur les points limites}

Définition 1. Soit $\Gamma$ un groupe localement compact et non compact opérant continûment sur un espace topologique $E$; on suppose $E$ et $\Gamma$ séparables. Pour $x \in E$ on appellera ensemble limite de $x$ sous $\Gamma$, l'ensemble $L_{x}$ des valeurs d'adhérence de l'orbite $\Gamma x$ suivant le filtre des complémentaires de parties compactes de $\Gamma$.

Il résulte de cette définition que $L_{x}$ est un fermé $\Gamma$-invariant. Un exemple typique est fourni par le cas où $\Gamma \subset S l(2, \mathbb{R})$ est engendré par 2 isométries hyperboliques du disque unité $D$ notées $\boldsymbol{A}$ et $\boldsymbol{B}$. Un polygone fondamental est dessiné ci-contre et on suppose que $A$ envoie l'extérieur de l'arc $a^{-1}$ sur l'intérieur de l'arc $a$; donc $A^{-1}$ envoie l'extérieur de $a$ sur l'intérieur de $a^{-1}$ et de même pour $B^{ \pm 1}$. On voit alors que les points $\gamma, 0(\gamma \in \Gamma)$ tendent vers un ensemble $C$ du type de Cantor sur le cercle unité dont on dessine ci-dessous l'approximation d'ordre 2 comportant $4 \times 3$ arcs disjoints. On a donc $C=L$.

Rappelons qu'une condition suffisante pour qu'une partie fermée d'un espace euclidien soit de dimension de Haussdorff $\geq \alpha$ est qu'elle porte une mesure $\nu$ vérifiant
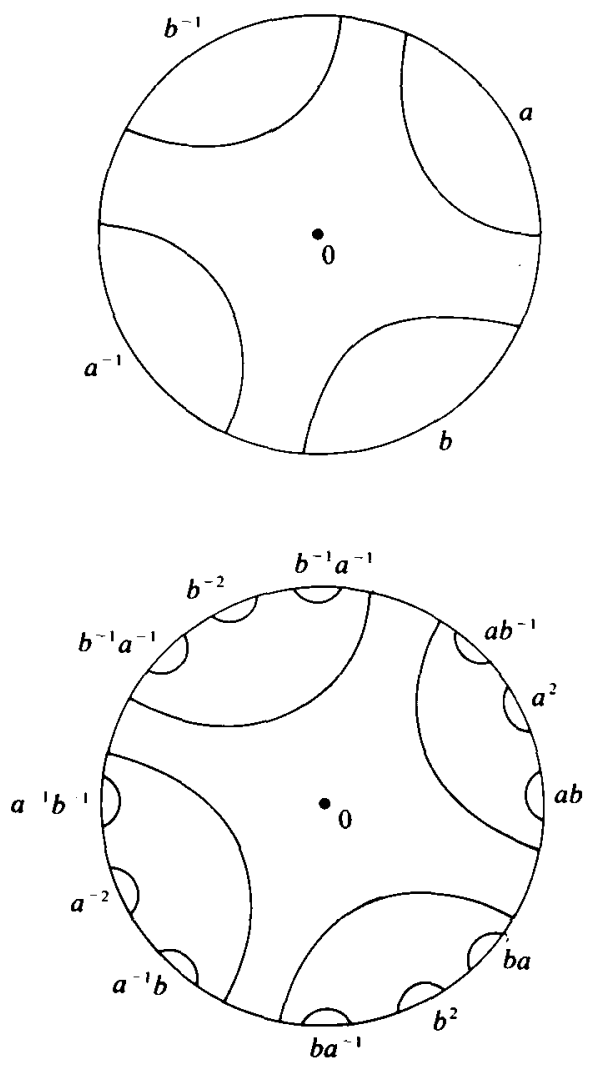
par toute boule $B(a, \varepsilon)$ centrée en $a \in A$ :

$$
\nu[B(a, \varepsilon)] \leq \text { cte } \varepsilon^{\alpha} .
$$

Il en est ainsi en particulier dès que $\operatorname{Sup}_{a \in A} \int 1 /\left(\|a-x\|^{\alpha} d \nu(x)<+\infty\right.$.

La construction d'une telle mesure $\nu$ est naturelle dans l'exemple précédent, ainsi que pour l'ensemble triadique de Cantor classique et on va dans la suite étendre cette construction, puis en tirer des conclusions géométriques.

\subsection{Frontieres, espaces symetriques, points limites}

La notion de proximalité ( $\$ 1.1$, Def. 1) jouera un rôle essentiel. Un exemple typique est fourni par l'action de $G=S l(d, \mathbb{R})$ sur l'espace projectif $P^{d-1}$. En effet, si $\gamma=\operatorname{diag}\left(a^{1}, \ldots, a^{d}\right)$ avec $a^{1}>a^{i+1}$ pour tout $i$ on voit que si $x \in P^{d-1}$ est choisi en dehors des sous-variétés projectives joignant un nombre fini des points $\overline{\boldsymbol{e}}_{i}$ associés aux vecteurs de base $e_{i}(1 \leq i \leq d)$, on $a: \lim _{n} \gamma^{n} x=\bar{e}_{1}$. La proximalité de $G$ sur $P^{d-1}$ découle alors de la transitivité de $G$ sur $P^{d-1}$. Plus généralement on rappelle [5] que la frontière de Fürstenberg $B$ de $G$ est formée de drapeaux, c'est-à-dire des suites de $d$ sous-espaces emboîtés distincts. Le drapeau canonique $e$ est, par exemple, défini par la suite de multivecteurs $\left(e_{1}, e_{1} \wedge e_{2}, \cdots e_{1} \wedge e_{2} \wedge \cdots \wedge e_{d}\right)$ correspondant à la suite des sous-espaces $\sum_{i<k} \mathbb{R} e_{i}$. L'argument précédent montre que pour $b \in B$ en dehors d'un sous-ensemble 'linéaire' exceptionnel on a $\lim _{n} \gamma^{n} b=e$, d'où encore la proximalité de l'action de $G$ sur $B$, justifiant selon [5] le terme de frontière de $G$.

Notons $A$ le groupe des matrices diagonales positives, $N$ celui des matrices triangulaires supérieures unipotentes, $K$ le groupe orthogonal de $\mathbb{R}^{d}, M$ le sousgroupe de $K$ formé des matrices diagonales à coefficients \pm 1 .

Comme le stabilisateur de $e$ est égal à $M A N$ on a: $B=G / M A N=K / M$ et la décomposition d'Iwasawa: $G=K A N$. En particulier $B$ est muni d'une mesure naturelle $K$-invariante que l'on notera $m$. Si alors $\gamma_{n}=\operatorname{diag}\left(a_{n}^{1}, \ldots, a_{n}^{d}\right)$ avec $a_{n}^{i+1}=$ $O\left(a_{n}^{i}\right)$, il est clair que $\lim _{n} \gamma_{n} m=\delta_{e}$ propriété qui sera utilisée de manière essentielle dans la suite.

Considérons l'espace symétrique $S=G / K$ qui s'identifie ici à l'espace des matrices symétriques définies positives de déterminant unité, l'action de $G$ sur $S$ étant définie par $g . M=g M g^{\prime}(g \in G, M \in S)$. Le noyau de Poisson $P(s, d b)$ fait correspondre à la matrice Id la mesure $m$ sur $B$. On notera ici 0 le point de $S$ défini par Id et $P(s, d b)$ fait alors correspondre au point $s=g .0$ la mesure $g . m$. Si $\mathscr{P}(B)$ désigne l'espace compact en topologie vague des mesures de probabilités sur $B$, on a ainsi un plongement bicontinu $s \rightarrow P(s,$.$) de s$ dans $\mathscr{P}(B)$. On notera $\bar{S}$ l'adhérence de cette image; elle est appelée classiquement compactification de Moore de $S$ [19] et on trouvera une description simple en [10]. Il est clair, vu la proximalité de $G$ sur $B$ que $\bar{S} \supset B$, où $B$ est ici identifié aux mesures de Dirac $\delta_{b}$ sur $B$. En général $\bar{S}$ est la réunion de l'ouvert $S$, du fermé $B$ et d'un nombre fini d'espaces homogènes de dimensions intermédiaires. Par exemple si $d=3$, les éléments de $\bar{S}-S \cup B$ sont de deux types correspondant à des mesures portées par $K$ identifié au fibré unitaire tangent à $S^{2}$ :

(1) Les mesures 'du type de Poisson' portées par les vecteurs tangents aux grands cercles de $S^{2}$. 
(2) Les mesures 'du type de Poisson' portées par le cercle des vecteurs unitaires tangents à $S^{2}$ en un point donné de $S^{2}$. Le stabilisateur d'une mesure du type 1 est de la forme

$$
\left(\begin{array}{ccc}
b(A) & a \\
0 & 0 & c
\end{array}\right)
$$

où $A$ est orthogonal et $c b^{2} \operatorname{det} A=1, a, b \in \mathbb{R}$. L'ensemble $S_{1}$ de ces mesures est donc un fibré en disques unités au-dessus d'un plan projectif $P^{2}$. Il en est de même de $S_{2}$ et l'on a $\bar{S}=S \cup B \cup S_{1} \cup S_{2}$ avec $B=\bar{S}_{1} \cap \bar{S}_{2}$ apparaissant comme une frontière commune de $S_{1}$ et $S_{2}$. Soit alors $\Gamma$ un sous-groupe fermé de $G, \sigma$ un point de $S$, considérons la partie de $S$ intersection de $B$ et $\overline{\Gamma \cdot \sigma}$ et notons $\lambda=P(\sigma, d b)$. Il est immédiat que la relation $\lim \gamma_{n} \cdot \lambda=\delta_{b}$ implique aussi $\lim \gamma_{n} \cdot m=\delta_{b}$. On peut donc poser la:

Définition 2. On appellera ensemble limite de $\Gamma$ la partie fermée de $B$ définie par $L_{1}=\overline{\Gamma \cdot 0} \cap B$.

On vient de voir que la proximalité de $\Gamma$ sur $B$ implique $L_{\Gamma} \neq \varnothing$. Pour une étude plus approfondie de $L_{1}$. on introduit la condition suivante.

Définition 3. On dira que $\Gamma$ est totalement irréductible sur $B$ s'il ne laisse pas invariante de réunion finie de sous-espaces de $\Lambda^{k} \mathbb{R}^{d}(1 \leq k \leq d-1)$.

Si $\Gamma$ est algébriquement dense la condition précédente est satisfaite. Il en est de même de la proximalité comme l'ont prouvé Goldsheid et G. A. Margulis [7].

Les premiers résultats de cette partie sont les suivants:

ThÉoRème 1. Supposons $\Gamma$ totalement irréductible et proximal sur B. Alors $\Gamma$ agit de manière minimale sur l'ensemble des points limites $L_{\Gamma^{\prime}}$. De plus si $L_{1} \neq B$ alors $L_{\Gamma^{\prime}}$ est parfait sans point intérieur.

ThÉORÈme 2. Dans la situation du Théorème $1, L_{\mathrm{I}}$ est de dimension de Hausdorff strictement positive.

La preuve sera donnée plus loin en utilisant les propriétés des produits de matrices aléatoires que l'on rappelle brièvement ci-dessous.

\subsection{Produits de matrices aleatoires}

Soit $\mu$ une mesure de probabilité sur $G$ de support $\sum_{\mu}, T_{\mu}$ (resp. $\Gamma_{\mu}$ ) le semi-groupe (resp. groupe) engendré par le support de $\mu$. L'action de $G$ sur $B$ définit une convolution entre mesures sur $G$ et $B$ : si $\mu$ et $\nu$ sont deux probabilités sur $G$ et $B$ respectivement on pose:

$$
\mu * \nu=\int_{B} \delta_{g \cdot h} d \mu(g) d \nu(b) .
$$

On notera $\mu^{\prime \prime}$ la $n^{\text {ième }}$ convolée de $\mu, \pi$ la mesure produit $\mu^{N}$ sur $\Omega^{+}=\left(\sum_{\mu}\right)^{N}$. Soient $X_{n}(\omega)$ les fonctions coordonnées sur $\Omega^{+}$et $S_{n}(\omega)$ le produit $S_{n}(\omega)=$ $X_{n}(\omega) X_{n-1}(\omega) \cdots X_{1}(\omega)$. 
Un résultat essentiel de $H$. Fürstenberg dit que si $T_{\mu}$ est irréductible et non compact on a:

$$
\begin{array}{cl}
\text { p.p. } & \lim _{n}(1 / n) \log \left\|S_{n}\right\|=\gamma_{1}>0 \\
\text { si } & \int \log \|g\| d \mu(g)<+\infty
\end{array}
$$

Concernant la frontière $B$ on a les théorèmes suivants $([5],[10])$.

THÉORÈme 3. Soit $\mu$ une mesure de probabilité sur $S l(d, \mathbb{R})$ telle que $T_{\mu}$ soit totalement irréductible et proximal sur B. Alors l'équation $\mu * \nu=\nu$ où $\nu$ est une probabilité sur $B$ a une solution unique et la suite $X_{1} \cdots X_{n}$. $\nu$ converge p.p. vers une mesure de Dirac.

ThÉorème 4. Avec les notations du Théorème 3 supposons de plus

$$
\int \log \|g\| d \mu(g)<+\infty
$$

et écrivons $S_{n}$ sous forme d'Iwasawa:

$$
S_{n}=K_{n} A_{n} N_{n} \quad \text { avec } \quad K_{n} \in K, N_{n} \in N, A_{n}=\operatorname{diag}\left(a_{n}^{1}, \ldots, a_{n}^{d}\right) \in A .
$$

On a alors p.p. et pour tout $i$

$$
\lim (1 / n) \log a_{n}^{1}=\gamma_{1}>0, \quad \lim (1 / n) \log a_{n}^{i} / a^{i+1}=\gamma_{i}-\gamma_{i+1}>0 .
$$

De plus $N_{n}$ converge p.p. et la projection de $K_{n}$ sur $B$ converge en loi.

ThÉorème 5. Avec les notations du Théorème 4 supposons de plus $\int\|g\|^{c} d \mu(g)<+\infty$ pour un certain $c>0$. Alors il existe $\varepsilon>0, \rho(0<\rho<1)$ et $C>0$ avec

$$
\int\left(a_{n}^{i+1} / a_{n}^{i}\right)^{\varepsilon}(\omega) d \pi(\omega) \leq C \rho^{n} .
$$

Afin d'interpréter ces résultats dans l'espace symétrique $S$ introduisons le drapeau $\mathrm{e}^{\prime}$ associé aux multivecteurs $\left(e_{d}, e_{d} \wedge e_{d-1}, \ldots, e_{d} \wedge e_{d-1} \wedge \cdots \wedge e_{1}\right)$. Posant $g_{i}=X^{-1}$, $N_{n}^{-1}=\eta_{n}, A^{-1}=\alpha_{n}$ on obtient: $g_{1} \cdots g_{n} \cdot m=\eta_{n} \alpha_{n} \cdot m$ et $\lim _{n} \alpha_{n} \cdot m=\delta_{e}$, donc $\lim \alpha_{n} \cdot 0=e^{\prime} . \lim \eta_{n}=\eta$ et $\lim g_{1} \cdots g_{n} \cdot 0=\eta \cdot e^{\prime}$. On a donc en particulier la convergence de la suite $g_{1} \cdots g_{n} \cdot 0$ vers un point de $\bar{S}-S$ situé dans $B$ et de loi $\nu^{\prime}$ vérifiant $\breve{\mu} * \nu^{\prime}=\nu^{\prime}$ où $\breve{\mu}$ est la symétrique de $\mu$. Le théorème suivant donne un type d'estimation essentiel pour l'étude du spectre d'un produit de matrices aléatoires.

Théorème 6. Avec les hypothèses du Théorème 3 et supposant de plus

$$
\int\|g\|^{\prime} d \mu(g)<+\infty
$$

pour un certain $c>0$, il existe $\varepsilon>0$ tel que $\int\|\eta(\omega)\|^{e} d \pi(\omega)<+\infty$.

On peut observer que la loi $\nu^{\prime}=\eta \cdot e^{\prime}$ peut être considérée comme la mesure harmonique de la marche aléatoire sur $\Gamma_{\mu}$ de trajectoires $g_{1} \cdots g_{n}$. 
Remarque. Les résultats de [13] montrent que l'hypothèse d'irréductibilité et de proximalité de $T_{\mu}$ ne dépend que de l'adhérence algébrique de $T_{\mu}$ qui est un groupe. Elle vaut donc pour $T_{\mu}^{-1}$ et $\left(T_{\mu}\right)^{\prime}$ aussi bien que pour $T_{\mu}$.

\subsection{Quelques propriétés de l'action de $\Gamma$ sur $L_{\Gamma}$}

ThÉORÈme 1. Supposons $\Gamma$ totalement irréductible et proximal sur $B$. Alors $\Gamma$ agit de manière minimale sur $L_{1}$. De plus si $L_{1} \neq B, L_{1}$ est parfait sans points intérieurs.

Preuve. Fixons une mesure $\mu$ symétrique avec $T_{\mu}=\Gamma$ que l'on peut supposer fermé. Montrons que le support $\sum_{\nu}$ de l'unique mesure invariante $\nu[\mu * \nu=\nu]$ est égal à $L_{\Gamma}$. Par construction de $\nu$, on a $\sum_{\nu} \subset L_{\Gamma}$. Si $x \in L_{\Gamma}$, il existe $\gamma_{n} \in \Gamma$ avec lim $\gamma_{n} \cdot m=\delta_{x}$. Mais la mesure $\nu$ possède des propriétés de non dégénérescence $([10],[12])$ et en particulier, comme on l'a vu ici ne charge pas le complémentaire de $N \cdot e^{\prime}$ dans $B$. Ceci suffit à assurer que l'on a aussi $\lim _{n} \gamma_{n} \cdot \nu=\delta_{x}$. Comme $\left(\gamma_{n} \cdot \nu\right)\left(\sum_{p}\right)=1$ puisque $\sum_{\nu}$ est invariant sous $T_{\mu}$ donc sous $\Gamma$ on a aussi à la limite: $\delta_{x} \in \sum_{\nu}$ donc $\sum_{\nu}=L_{1}$.

Si maintenant $F \subset L_{1}$. est un fermé invariant sous l'action de $\Gamma$ l'opérateur de convolution par $\mu$ sur les probabilités portées par $F$ admet un point fixe $\nu_{1}$ : $\mu * \nu_{1}=\nu_{1}$. Comme il y a unicité de la mesure $\mu$-invariante d'après le Théorème 3 , on a $\nu_{1}=\nu$ et $F=L_{1}=\sum_{\nu}$. Donce $\Gamma$ est minimal sur $L_{1}$. En particulier $L_{1}$ est égal à l'ensemble $D$ de ses points d'accumulation puisque celui-ci est un fermé $\Gamma$ invariant. De même si $L_{\mathrm{l}} \neq B$, la frontière $L_{1}-L_{\mathrm{l}}^{0}$ est non vide et est un fermé $\Gamma$-invariant: par minimalité $L_{\mathrm{\Gamma}}-L_{\mathrm{l}}^{0}=L_{\mathrm{l}}$, ce qui donne $L_{\mathrm{l}}^{0}=\varnothing$.

Le recours à l'espace symétrique $S$ n'est pas nécessaire pour la définition des points limites. On peut en effet faire opérer directement $\Gamma$ sur la frontière de Fürstenberg $B$ ou même sur des sous-frontières telles que l'espace projectif $P^{d-1}$. Ceci permet de donner la.

Définition 4. Soit $\Gamma$ un sous-groupe de Sl $(d, \mathbb{R})$. On appelle point limite de $\Gamma$ sur $P^{d-1}$ tout point $p \in P^{d-1}$ tel qu'il existe une suite $\gamma_{n} \in \Gamma$ tendant vers l'infini avec $\lim \gamma_{n} . m=\delta_{n}$ où $m$ désigne la mesure invariante par rotation sur $P^{d-1}$. L'ensemble de ces points limites sera noté $L_{1}^{\prime}$. Il est facile de voir que l'ensemble $L_{1}^{\prime}$ des points limites est un fermé $\Gamma$-invariant.

On a alors l'analogue du Théorème 1:

THÉORĖme 1'. Soit $\Gamma$ un sous-groupe de $\mathrm{Sl}(d, \mathbb{R})$ opérant de manière totalement irréductible et proximale sur $P^{d-1}$. Alors l'ensemble des points limites $L_{1}$ est un fermé non vide sur lequel $\Gamma$ agit de manière minimale. Si $L_{1}^{1} \neq P^{d-1}, L_{1}^{1}$ est parfait sans points intérieurs. De plus $L_{1}^{1}$ est l'adhérence de l'ensemble des points attractifs des éléments de $\Gamma$ ayant une valeur propre dominante simple.

Preuve. Il suffit d'utiliser l'analogue du Théorème 3 sous l'hypothèse affaiblie qui assure encore des résultats analogues si l'on remplace $B$ par $P^{d-1}[10]$. Le fait que $L_{1}^{1}$. soit non vide découle de [5]. Dans la partie 2.1 on a vu au Théorème 3 que $\Gamma$ contenait des éléments ayant une valeur propre dominante simple; l'ensemble des points attractifs correspondants est donc non vide et contenu dans $L_{1}^{i}$. Son adhérence est un fermé $\Gamma$-invariant qui est égal à $L_{\Gamma}^{1}$ par minimalité de l'action de $\Gamma$ sur $L_{1}^{1}$. On a aussi les analogues des Théorèmes $3,4,5$ qui seront brièvement justifiés. 
Avant de montrer le Théorème 2, prouvons le.

THÉORÈME $2^{\prime}$. Soit $\Gamma$ un sous-groupe de $\mathrm{Sl}(d, \mathbb{R})$ opérant de manière totalement irréductible et proximale sur $P^{d-1}$. Alors l'ensemble des points limites $L_{1}^{1}$ est de dimension de Hausdorff strictement positive.

Le Théorème 2 découle immédiatement du Théorème $2^{\prime}$ et du lemme suivant car la projection de $B$ sur $P^{d-1}$ est Lipchitzienne pour les métriques naturelles.

Lemme 1. Dans la situation du Théorème 1 , la projection naturelle de $B$ sur $P^{d-1}$ applique $L_{\mathrm{N}}$ sur $L_{1}^{1}$.

Preuve. Par définition de $L_{\Gamma}$ et $L_{\Gamma}^{1}$ la projection de $L_{\Gamma}$. dans $P^{d-1}$ est un compact $\Gamma$-invariant contenu dans $L_{1}^{1}$. Par minimalité de l'action de $\Gamma$ sur $L_{1}^{1}$, ce compact est égal à $L_{\mathrm{K}}^{\prime}$.

La preuve du Théorème 2 ' repose sur le.

LeMme 2. Soit $(C, d)$ un espace métrique compact, $A$ et $B$ deux compacts disjoints de $C$, s et $t$ deux applications continues de $C$ dans $C$ vérifiant $s(C) \subset A, t(C) \subset B$.

On suppose que pour deux constantes $\left.\rho, \rho^{\prime} \in\right] 0,1[$ on a

$$
\begin{array}{ll}
\forall x, y \in C \quad & \rho^{\prime} d(x, y) \leq d(s x, s y) \leq \rho d(x, y) \\
& \rho^{\prime} d(x, y)<d(t x, t y) \leq \rho d(x, y) .
\end{array}
$$

Alors, notant $S=\{s, t\}$ et $C^{\propto}=\bigcap_{n>0} S^{n} C$, le compact $C^{\infty}$ est homéomorphe à l'espace produit $S^{N}$ et de dimension de Hausdorff strictement positive.

Preuve. Soit $\sigma=\left(s_{k}\right)_{k \in N} \in S^{N}$ et observons que la suite $s_{1} s_{2} \cdots s_{n} C$ est décroissante de diamètre majoré par $c \rho^{n}$; son intersection définit donc un point $\bar{\sigma}=$ $\lim _{n} s_{i} s_{n} \cdots s_{n} C$. Comme d'autre part $S^{n} C$ est réunion disjointe des $s_{1} s_{2} \cdots s_{n} C$ $\left(s_{1} \in S\right)$, il est clair que $C^{x}$ est l'image de $S^{N}$ par l'application $\sigma \rightarrow \bar{\sigma}$. Soient $\sigma$, $\sigma^{\prime} \in S^{N}$ avec $\sigma=\left(s_{k}\right), \sigma=\left(s_{k}^{\prime}\right)$ et $s_{k}=s_{k}^{\prime}(k \leq n)$ et estimons $d\left(\bar{\sigma}, \bar{\sigma}^{\prime}\right)$. Pour $x \in C$ on a $\bar{\sigma}=\lim _{k} s_{1} s_{2} \cdots s_{k} x, \bar{\sigma}^{\prime}=\lim _{k} s_{1}^{\prime} s_{2}^{\prime} \cdots s_{k}^{\prime} x$.

Or $d\left(s_{n+1} \cdots s_{k} x, s_{n+1}^{\prime} \cdots s_{k}^{\prime} x\right) \leq c$ et donc $d\left(\bar{\sigma}, \bar{\sigma}^{\prime}\right) \leq c \rho^{n}$.

De même, puisque $s_{n+1} \neq s_{n+1}^{\prime}$ on a

$$
d\left(s_{n+1} \cdots s_{k} x, s_{n+1}^{\prime} \cdots s_{k}^{\prime} x\right) \geq d(A, B)=\varepsilon>0
$$

et $d\left(\bar{\sigma}, \bar{\sigma}^{\prime}\right) \geq \rho^{\prime n} \varepsilon$.

L'espace $S^{N}$ muni de la métrique définie par $\delta\left(\sigma, \sigma^{\prime}\right)=1 / 2^{n}$ pour $\sigma$ et $\sigma^{\prime}$ du type précédent est compact, de dimension de Hausdorff 1 . Les inégalités précédentes peuvent alors s'écrire avec des constantes $\alpha, \beta>0$

$$
\varepsilon \delta\left(\sigma, \sigma^{\prime}\right)^{\beta} \leq d\left(\bar{\sigma}, \bar{\sigma}^{\prime}\right) \leq c \delta\left(\sigma, \sigma^{\prime}\right)^{\alpha} .
$$

On a donc l'homéomorphisme de $C^{\infty}$ et $S^{N}$ avec positivité de la dimension de Hausdorff de $C^{\alpha}$, majorée en fonction de $\alpha$ et minorée en fonction de $\beta$.

LEM ME 3. Soient $g$ et $h$ deux applications linéaires ayant des valeurs propres dominantes simples, réelles. On suppose le point attractif de $g$ distinct de celui de $h, n$ 'appartenant pas à l'hyperplant répulsif de $h$ et vice-versa. Alors, pour la métrique naturelle $\delta$ sur $P^{d-1}$, il existe deux boules fermées disjointes $A$ et $B$, un entier $n>0$ et des constantes 
$\left.\rho, \rho^{\prime} \in\right] 0,1[$ telles que

$$
\begin{gathered}
g^{n}(A \cup B) \subset A, \quad h^{n}(A \cup B) \subset B \\
\rho^{\prime} \delta(x, y) \leq \delta(r x, r y) \leq \rho \delta(x, y)
\end{gathered}
$$

pour $x, y \in A \cup B$ et $r=g^{n}$ ou $h^{n}$.

Preuve. Soient 0 un point de $P^{d-1}, H$ un hyperplan, $u$ une application projective fixant 0 et $H$. On peut alors considérer $P^{d-1}-H$ comme un espace vectoriel $\mathbb{R}^{d-1}$, que l'on peut normer, et $u$ comme une application linéaire inversible. Si $u$ admet une valeur propre dominante simple, 0 étant le point attractif correspondant, on a pour tout compact $C$ de $P^{d-1}-H: \lim _{n} u^{n}(C)=0$. Donc pour $n$ assez grand il existe $\eta, \varepsilon \in] 0,1[$ avec

$$
\varepsilon\|x\| \leq\left\|u^{n} x\right\| \leq \eta\|x\| \quad(x \notin H) .
$$

Pour le compact $C$, il existe d'autre part deux constantes $a$ et $b$ telles que sur $C: a\|x-y\| \leq \delta(x, y) \leq b\|x-y\|$. Le compact $C$ étant choisi de façon que $u(C) \subset C$, on en déduit pour tout $p \in \mathbb{N}$ et $x, y$ dans $C$ :

$$
\frac{a}{b} \varepsilon^{p} \delta(x, y) \leq \delta\left(u^{n p} x, u^{n p} y\right) \leq \frac{b}{a} \eta^{p} \delta(x, y) .
$$

D'où en prenant $p$ assez grand, l'existance de $\rho, \rho^{\prime} \in[0,1[$, avec

$$
\forall(x, y) \in C \times C: \rho^{\prime} \delta(x, y) \leq \delta\left(u^{n p} x, u^{n p} y\right) \leq \rho \delta(x, y) .
$$

Revenant à la situation du lemme prenons pour $A$ et $B$ des boules centrées aux points attractifs de $g$ et $h$, ne rencontrant pas les hyperplans répulsifs. Alors pour tout $n$ assez grand $g^{n}(A \cup B) \subset A, h^{n}(A \cup B) \subset B$. Utilisant le début de la preuve avec $u=g^{n}$ ou $h^{n}, C=A \cup B$ on obtient, en prenant $n$ assez grand $\left(r=g^{n}\right.$ ou $\left.h^{n}\right)$ $\left.\rho, \rho^{\prime} \in\right] 0,1\left[\right.$ avec $\rho^{\prime} \delta(x, y) \leq \delta(r x, r y) \leq \rho \delta(x, y)$ pour $(x, y) \in C \times C$.

Preuve $d u$ Théorème $2^{\prime}$. Le Théorème 3 de la partie I permet de trouver $g$ et $h$ dans $\Gamma$ ayant des valeurs propres dominantes simples, à cause de la proximalité et de l'irréductibilité de l'action de $\Gamma$ sur $P^{d-1}$. Les Lemmes 2 et 3 montrent alors, avec $s=g^{n}, t=h^{n}, S=\{s, t\}$ qu'il existe $C$ d'intérieur non vide tel que le compact $C^{\infty}=\bigcap_{n} S^{n} C$ soit de dimension de Hausdorff positive. Si $m_{1}$ est la restriction de la mesure de Lebesgue à $C$ et $\sigma=\left(s_{k}\right) \in S^{N}$; la suite de mesures $s_{1} \cdots s_{k} \bar{m}$ converge vers la mesure de Dirac en $\bar{\sigma}=\bigcap_{k}\left(s_{1} \cdots s_{k} C\right)$. Il en est donc de même de la suite $s_{1} \cdots s_{k} m[9]$ et $\bar{\sigma} \in C^{\infty}$ est un point limite de $\Gamma$. Cet ensemble de points limites $L_{1}^{1}$. contient donc $C^{\alpha}$ et est de dimension de Hausdorff positive.

\subsection{Calculs de moments sur les groupes nilpotents}

Le groupe $N$ présente une structure graduée facilitant les calculs. En effet si $E_{i j}$ est la matrice dont tous les coefficients sont nuls à l'exception du terme dans la $i^{i e ̀ m e}$ ligne et $j^{\text {ième }}$ colonne où il vaut 1 , on peut décomposer l'algèbre de Lie $\eta$ de $N$ sous la forme

$$
\eta=\bigoplus_{i=1}^{d-1} \eta^{i} \quad \text { avec } \quad \eta^{i}=\sum_{k-j=i} \mathbb{R} \cdot E_{j k}
$$

et les $\eta^{i}$ définissent une graduation de $\eta$. On identifiera $\eta$ et $N$ par l'exponentielle, le produit $x \circ y(x, y \in N)$ étant alors donné par la formule de Campbell-Hausdorff. 
Les éléments $\alpha$ de $A$ définissent une famille naturelle commutative d'automorphisme de $N: \alpha(\beta)=\alpha \beta \alpha^{-1}(\beta \in N)$. En particulier on notera $\Lambda$ la famille 'd'homothéties' $\alpha=\operatorname{diag}\left(\lambda^{d}, \lambda^{d-1}, \ldots, \lambda\right)$; l'action d'un tel $\alpha$ sur $\eta^{i}=N^{i}$ se réduit à la multiplication par $\lambda^{i}$. Ayant fait des choix de normes sur les espaces vectoriels $\eta^{i}: \beta^{i} \rightarrow\left|\beta^{i}\right|$, on peut 'normer' $\eta=N \operatorname{par}((\beta))=\operatorname{Sup}_{1 \leq i \leq d-1}\left|\beta_{i}\right|^{1 / i}$ où $\beta=\sum_{i=1}^{d-1} \beta_{i}$. Alors, si l'on note $u_{\lambda}$ l'élément de $\Lambda$ définie par $\lambda$ on a [11]

$$
\begin{aligned}
\left(\left(u_{\lambda}(x)\right)\right) & =|\lambda|((x)) \quad(x \in N) \\
((x \circ y)) & \leq((x))+((y))+\text { cte. }
\end{aligned}
$$

Ici à cause de la graduation la constante est nulle:

$$
\left(\left(u_{\lambda}(x \circ y)\right)\right)=\left(\left(u_{\lambda}(x) \circ u_{\lambda}(y)\right)\right) \leq|\lambda|((x))+|\lambda|((y))+\text { cte }
$$

et aussi $\left(\left(u_{\lambda}(x \circ y)\right)\right)=|\lambda|((x \circ y))$. Donc

$$
((x \circ y)) \leq((x))+((y))+(\text { cte } /|\lambda|)
$$

et prenant $|\lambda|$ grand, on obtient bien $((x \circ y)) \leq((x))+((y))$. Pour un automorphisme $u$ de $N$ commutant avec $\Lambda$ on peut alors définir une 'norme' par

$$
((u))=\sup _{x \neq 0}((u x)) /((x))=\operatorname{Sup}_{((x))=1}((u x)) .
$$

En particulier si $\alpha=\operatorname{diag}\left(\alpha^{1}, \alpha^{2}, \ldots, \alpha^{d}\right)$ on voit que la norme de l'automorphisme associé à $\alpha$ est $((\alpha))=\operatorname{Sup}_{1 \leq i \leq d}\left|\alpha^{i} / \alpha^{i+1}\right|$.

Il est clair que $N$ est complet pour la distance associée à $((\cdot))$ et il est donc aisé d'étendre un certain nombre de propriétés de $\mathbb{R}^{n}$.

Lem ME 4. Soient $\alpha_{n}(\omega), \beta_{n}(\omega)$ des suites aléatoires d'éléments de A, N respectivement, vérifiant pour un certain $\varepsilon>0$.

$$
\begin{gathered}
\varlimsup_{n}\left[\int\left(\left(\alpha_{n}(\omega)\right)\right)^{\varepsilon} d \pi(\omega)\right]^{1 / n}=\rho<1, \\
\operatorname{Sup}_{n} \int\left(\left(\beta_{n}(\omega)\right)\right)^{\varepsilon} d \pi(\omega)<+\infty
\end{gathered}
$$

Alors le produit

$$
\eta_{n}=\beta_{1} \circ \alpha_{1}\left(\beta_{2}\right) \circ \alpha_{2}\left(\beta_{3}\right) \circ \cdots \circ \alpha_{n-1}\left(\beta_{n}\right)
$$

converge p.p. vers $\eta$ et l'on a

$$
\int((\eta(\omega)))^{F / 2} d \pi(\omega)<+\infty, \varlimsup_{n}\left[\int\left(\left(\eta_{n}^{-1} \eta(\omega)\right)\right)^{\varepsilon / 2} d \pi(\omega)\right]^{1 / n}=\rho<1 .
$$

Preuve. On va appliquer le critère de Cauchy à la suite $\eta_{n}$

$$
\begin{aligned}
\left(\left(\eta_{n}\right)\right) & \leq \sum_{i=1}^{n-1}\left(\left(\alpha_{i}\right)\right)\left(\left(\beta_{i+1}\right)\right), \\
\left(\left(\eta_{n}\right)\right)^{F / 2} & \leq \sum_{i=1}^{n-1}\left(\left(\alpha_{i}\right)\right)^{F / 2}\left(\left(\beta_{i+1}\right)\right)^{F / 2}, \\
\int\left(\left(\eta_{n}\right)\right)^{F / 2} d \pi & \leq \sum_{i=1}^{n-1}\left(\int\left(\left(\alpha_{i}\right)\right)^{F} d \pi\right)^{1 / 2}\left(\int\left(\left(\beta_{i+1}\right)\right)^{F} d \pi\right)^{1 / 2}, \\
\int\left(\left(\eta_{n}\right)\right)^{r / 2} d \pi & \leq \operatorname{cte}\left(1 / 1-\rho^{1 / 2}\right)<+\infty .
\end{aligned}
$$


De la même façon

$$
\left(\left(\eta_{n}^{-1}\right) \eta_{n+p}\right) \leq \sum_{n}^{\infty}\left(\left(\alpha_{i}\right)\right)\left(\left(\beta_{i+1}\right)\right)
$$

Posant $U_{n}=\operatorname{Sup}_{p}\left(\left(\eta_{n}^{-1}\right) \eta_{n+p}\right)$ on obtient

$$
\int U_{n}(\omega)^{\kappa / 2} d \pi(\omega) \leq \operatorname{cte} \rho^{n / 2}
$$

Ceci prouve d'une part la convergence presque sûre de $U_{n}(\omega)$ vers zéro, donc la convergence de $\eta_{n}$ vers $\eta$. On a donc

$$
\int\|\eta(\omega)\|^{\varepsilon / 2} d \pi(\omega)<+\infty
$$

De plus:

$$
\int\left\|\eta_{n}^{-1} \eta(\omega)\right\|^{\varepsilon / 2} d \pi(\omega) \leq \int U_{n}(\omega)^{\varepsilon / 2} d \pi(\omega) \leq \text { cte } \rho^{n / 2}
$$

LeMme 5. Soit $g_{i}(1 \leq i \leq n)$ une suite d'éléments de $\mathrm{Sl}(d, \mathbb{R})$ et décomposons les produits $g_{1} \cdots g_{i}$ sous la forme d'Iwasawa: $g_{1} \cdots g_{1}=\eta_{i} \alpha_{i} k_{i}\left(\eta_{i} \in N, \alpha_{i} \in A, k_{i} \in K\right)$. Alors $\eta_{n}$ s'écrit avec des $\beta_{i}$ fonctions de $g_{i}$ et $k_{i-1}$ :

$$
\eta_{n}=\beta_{1} \circ \alpha_{1}\left(\beta_{2}\right) \circ \cdots \circ \alpha_{n-1}\left(\beta_{n}\right) .
$$

Preuve. On l'établit par récurrence sur $n$. Notons $k g=b(g, k)(k \circ g)$ où $k \circ g \in K=$ $G / A N, a(g, k) \in N$ et admettons la relation voulue à l'ordre $n$. Alors, à l'ordre $n+1$ :

$$
g_{1} \cdots g_{n} g_{n+1}=\eta_{n} \alpha_{n} b\left(g_{n+1} k_{n}\right) a\left(g_{n+1}, k_{n}\right) k_{n} \cdot g_{n+1}
$$

d'où en particulier

$$
\eta_{n+1}=\eta_{n} \circ \alpha_{n}\left(\beta_{n+1}\right) \text { avec } \beta_{n+1}=b\left(g_{n+1}, k_{n}\right) .
$$

De plus on note que $\alpha_{n+1}=\alpha_{n} a\left(g_{n+1}, k_{n}\right), k_{n+1}=k_{n} \cdot g_{n+1}$.

Preuve du Théorème 6. Considérons maintenant la situation du Théorème 6 avec $g_{1} \cdots g_{n}=\eta_{n} \alpha_{n} k_{n}$ et les $g_{i}(\omega) \in \sum_{\mu}$ satisfaisant $\int\left\|g_{i}(\omega)\right\|^{c} d \pi(\omega) \leq$ cte.

Le Lemme 4 donne l'écriture de $\eta_{n}$ sous la forme $\eta_{n}=\prod_{i=1}^{n-1} \alpha_{i-1}\left(\beta_{i}\right)$ avec des $\beta_{i}$ vérifiant comme les $g_{i}$ :

$$
\int\left\|\beta_{i}(\omega)\right\|^{c} d \pi(\omega) \leq \text { cte. }
$$

Observons que les calculs d'exponentielles et de logarithme dans $N$ donnent

$$
((\beta)) \leq \operatorname{cte}\left(1+\|\beta\|^{d-1}\right), \quad\|\beta\| \leq \operatorname{cte}\left[1+((\beta))^{d-1}\right], \quad\|\beta-I\| \leq \operatorname{cte} \sum_{1}^{d^{2}}((\beta))^{k}
$$

on a donc aussi

$$
\int\left(\left(\beta_{i}(\omega)\right)\right)^{c^{\prime}} d \pi(\omega) \leq \mathrm{cte} \text { avec } c^{\prime}=c /(d-1)
$$


Comme d'autre part $\left(\left(\alpha_{p}\right)\right)=\operatorname{Sup}_{1<j<d-1}\left|a^{j+1} / a^{j}\right|$ le Théorème 5 relatif aux produits de matrices aléatoires nous donne

$$
\lim _{n}\left[\int\left(\left(\alpha_{n}(\omega)\right)\right)^{F^{\prime}} d \pi(\omega)\right]^{1 / n} \leq \rho<1
$$

pour un certain $\varepsilon^{\prime}$. On peut donc appliquer le Lemme 1 qui donne si $\varepsilon^{\prime}<c /(d-1)$ :

$$
\int((\eta(\omega)))^{\varepsilon^{\prime} / 2} d \pi(\omega)<+\infty, \quad \varlimsup\left[\int\left(\left(\eta_{n}^{-1} \eta(\omega)\right)\right)^{f^{\prime} / 2} d \pi(\omega)\right]^{1 / n}<1 .
$$

Enfin la comparaison de $((\eta))$ et $\|\eta\|$ donne $\int\|\eta(\omega)\|^{*} d \pi(\omega)<+\infty$ avec $\varepsilon=$ $\varepsilon^{\prime} / 2(d-1)$. La relation $\left\|\eta_{n}-\eta\right\| \leq\left\|\eta_{n}\right\|\left\|I-\eta_{n}^{-1} \eta\right\|$ permet d'aboutir de même $a$ :

$$
\varlimsup\left[\int\left\|\eta_{n}-\eta\right\|^{\lambda} d \pi(\omega)\right]^{1 / n}<1 \text { avec } \lambda=\varepsilon^{\prime} / 2 d^{2}
$$

\subsection{Estimation de la mesure invariante sur la frontiere de fürstenberg}

On a vu que la frontière $B$ s'identifie à $K / M$; d'autre part $K$ est muni de la distance de la norme $d\left(k, k^{\prime}\right)=\left\|k-k^{\prime}\right\|$; une distance naturelle $\delta$, invariante sur $K$ se trouve donc définie sur $B$ par passage au quotient. Pour $b$ et $b^{\prime}$ dans $B$ on peut en particulier trouver des représentants $k$ et $k^{\prime}$ dans $k$ tels que: $\delta\left(b, b^{\prime}\right)=\left\|k-k^{\prime}\right\|$.

THÉORÈME 7. Soit $\mu$ une mesure sur $\operatorname{Sl}(d, \mathbb{R})$ vérifiant $\int\|g\|^{c} d \mu(g)<+\infty$ pour un certain $c>0$ telle que $T_{\mu}$ soit totalement irréductible et proximal sur $B$. Soit $\nu^{\prime}$ la loi de $\lim g_{1} \cdots g_{n} \cdot 0 \in B$. On a alors $\operatorname{Sup}_{b \in B} \int\left(1 / \delta^{*}\left(b, b^{\prime}\right)\right) d \nu^{\prime}\left(b^{\prime}\right)<+\infty$ pour un certain $\varepsilon>0$.

Preuve. On a vu, avec les notations précédentes que $\lim _{n} g_{1} \cdots g_{n} \cdot 0=\eta \cdot e^{\prime} \in$ $N \cdot e^{\prime} \subset B$ et que $\int\|\eta(\omega)\|^{f} d \pi(\omega)<+\infty$. Soit $w$ un élément de $K$ avec $w e^{\prime}=e, k$ un élément de $K$ correspondant à $\eta$ et donc défini par $\eta \cdot e^{\prime}=k \cdot e^{\prime}$. On peut observer que $k$ est défini modulo $M$ et que l'application $\eta \rightarrow \bar{k} \in K / M$ est l'analogue de la projection stéréographique classique. Afin de comparer $\|k-w\|$ et $\|\eta\|$ notons que: $\left(k e_{d}\right)\left\|\eta e_{d}\right\|= \pm \eta e_{d}$ et donc $\left|\left\langle k e_{d}, e_{d}\right\rangle\right|\left\|\eta e_{d}\right\|=1$, soit $\|\eta\|\left|\left\langle k e_{d}, e_{d}\right\rangle\right| \geq 1$.

Or si

$$
w e_{d}= \pm e_{1}
$$

on a

$$
\left|\left\langle k e_{d}, e_{d}\right\rangle\right|=\left|<(k-w) e_{d}, e_{d}\right\rangle \mid \leq\|k-w\|
$$

ce qui donne $\|k-w\| \geq\left|\left\langle k e_{d}, e_{d}\right\rangle\right| \geq(1 /\|\eta\|)$. On a donc bien, d'après le Théorème 5 , en projetant dans $B$ :

$$
\int\left(1 / \delta^{\varepsilon}(\bar{w}, \bar{k})\right) d \nu(\bar{k})<+\infty
$$

Si $r \in K$, le changement de $y$ par $\delta_{r} * \mu * \delta_{r-1}$ aboutit à changer $\nu^{\prime}$ en $r \cdot \nu^{\prime}$ et l'uniformité valable dans le Théorème 5 relatif aux matrices aléatoires donne en particulier des $\varepsilon$ et $\rho$ valable pour tout $r$ et donc

$$
\int\left(1 / \delta^{f}(\bar{w}, b)\right) d r \cdot \nu^{\prime}(b) \leq+\infty
$$


comme $\delta(r \cdot \bar{w}, r \cdot b)=\delta(\bar{w}, b)$ on obtient, pour tout $r \in B$ :

$$
\int\left(1 / \delta^{\varepsilon}(r \cdot \bar{w}, b)\right) d \nu^{\prime}(b) \leq \text { cte }<+\infty
$$

ce qui achève la preuve car $K$ est transitif sur $B$.

Remarque. Ce théorème fournit une nouvelle preuve du Théorème 2.

\subsection{Estimation de la mesure invariante sur l'espace projectif}

ThÉORÈME 7'. Avec l'hypothèse du Théorème 2', soit $\mu$ une mesure de probabilité sur $\Gamma$ telle que $T_{\mu}=\Gamma$ et $\int\|g\|^{c} d \mu(g)<+\infty$ pour un certain $c>0, \nu$ l'unique mesure $\mu$-invariante sur $P^{d-1}(\mu * \nu=\nu)$. Alors pour la distance naturelle $\delta$ sur $P^{d-1}$ et le produit scalaire $\langle a, b\rangle$ sur $S^{d-1}$ on a

$$
\begin{gathered}
\operatorname{Sup}_{\bar{a} \in P^{d-1}} \int\left(1 / \delta^{f}(\bar{a}, \bar{b})\right) d \nu(\bar{b})<+\infty \text { pour un certain } \varepsilon>0 . \\
\operatorname{Sup}_{\bar{a} \in P^{d-1}} \int \frac{1}{|\langle a, b\rangle|^{f}} d \nu(\bar{b})<+\infty .
\end{gathered}
$$

Afin de justifier cet énoncé ou plutôt un énoncé équivalent, donnons quelques notations et lemmes. Si $N$ est le groupe des matrices triangulaires supérieures unipotentes, $V$ le sous-groupe de $N$ vérifaint $V e_{i}=e_{i}(1 \leq i \leq d-1), M$ le sousgroupe de $N$ vérifiant $M e_{d}=e_{d}$, on a la décomposition en produit semi-direct $N=V \cdot M$ où $V$ est distingué et s'identifie à l'espace vectoriel $\mathbb{R}^{d-1}$. Ainsi $N$ apparaît comme un sous-groupe du groupe affine de $\mathbb{R}^{d-1}$ et $P^{d-1}$ comme l'espace projectif complété de l'espace affine $V e_{d} \sim \mathbb{R}^{d-1}$. Les éléments de $M$ s'identifient à des matrices $(d-1 \times d-1)$ et sont donc naturellement normés.

LeMME 6. Soient $m_{n}(\omega), v_{n}(\omega)$ des suites aléatoires d'éléments de $M, V$ vérifiant pour un certain $\varepsilon \in] 0,1[$ :

$$
\begin{aligned}
& \varlimsup_{n}\left[\int\left\|v_{n}(\omega)\right\|^{\varepsilon} d \pi(\omega)\right]^{1 / n}=\rho<1 \\
& \varlimsup_{n}\left[\int\left\|m_{n}(\omega)\right\|^{\varepsilon} d \pi(\omega)\right]^{1 / n}=1 / \rho .
\end{aligned}
$$

Alors la composante $s_{n}$ sur $V$ du produit $\prod_{n}=v_{1} m_{1} v_{2} m_{2} \cdots v_{n} m_{n}$ converge p.p. vers $s((\omega))$ et l'on $a$ :

$$
\int\|s(\omega)\|^{F^{\prime}} d \pi(\omega)<+\infty, \quad \varlimsup_{n}\left[\int\left\|s(\omega)-s_{n}(\omega)\right\|^{F^{\prime}} d \pi(\omega)\right]^{1 / n}<1
$$

pour $\varepsilon^{\prime}=\left(\varepsilon / 2(d-2)^{2}\right)$.

Preuve. On écrit $s_{n}(\omega)$ composante de $\prod_{n}(\omega)$ :

$$
s_{n}=v_{1}+m_{1}\left(v_{2}\right)+m_{1} m_{2}\left(v_{3}\right)+\cdots+m_{1} \cdots m_{n-1}\left(v_{n}\right)
$$

et

$$
\left\|s_{n}\right\|^{r^{\prime}} \leq \sum_{k}\left\|m_{1} \cdots m_{k}\right\|^{k^{\prime}}\left\|v_{k+1}\right\|^{r^{\prime}}
$$


On a vu précédemment que pour $m \in M$

$$
\|m\| \leq 1+\operatorname{cte}((m))^{d-2}, \quad((m)) \leq \operatorname{cte}\left[1+\|m\|^{d-1}\right] .
$$

On en déduit:

$$
\begin{aligned}
& \left\|m_{1} \cdots m_{k}\right\|^{F^{\prime}} \leq 1+\operatorname{cte}\left[\left(\left(m_{1}\right)\right)^{(d-2) \varepsilon^{\prime}}+\cdots+\left(\left(m_{k}\right)\right)^{(d-2) \varepsilon^{\prime}}\right] \\
& \left\|m_{1} \cdots m_{k}\right\|^{\varepsilon^{\prime}} \leq 1+\operatorname{cte}\left[+\left\|m_{1}\right\|^{\varepsilon / 2}+\cdots+\left\|m_{k}\right\|^{\varepsilon / 2}\right] .
\end{aligned}
$$

L'hypothèse nous donne que pour $\varepsilon^{\prime \prime}$ assez petit et un certain $t>0$ :

$$
\begin{aligned}
& \int\left\|v_{n}(\omega)\right\|^{\varepsilon} d \pi(\omega) \leq \operatorname{cte}\left(\rho+\varepsilon^{\prime \prime}\right)^{n} \\
& \int\left\|m_{n}(\omega)\right\|^{\varepsilon} \mathrm{d} \pi(\omega) \leq \operatorname{cte}\left[1 / \rho-t+\varepsilon^{\prime \prime}\right]^{n}
\end{aligned}
$$

On en déduit, par l'inégalité de Schwarz,

$$
\int\left\|m_{1} \cdots m_{k}\right\|^{\varepsilon^{\prime}}\left\|v_{k+1}\right\|^{\varepsilon^{\prime}} d \pi(\omega) \leq \operatorname{cte}\left(\rho+\varepsilon^{\prime \prime}\right)^{k}\left[1+k+k\left((1 / \rho)-t+\varepsilon^{\prime \prime}\right)^{k}\right] .
$$

Si $\varepsilon^{\prime \prime}$ est choisi de façon que $\rho+\varepsilon^{\prime \prime}<1$ et $\left.\left(\rho+\varepsilon^{\prime \prime}\right)\left((1 / \rho)-t+\varepsilon^{\prime \prime}\right)-t+\varepsilon^{\prime \prime}\right)<1$, on a obtenu la convergence géométrique de la série des intégrales des termes $\left\|m_{1} \cdots m_{k}\right\|^{\varepsilon^{\prime}}\left\|v_{k+1}\right\|^{F^{\prime}}$ et donc, comme précédemment la convergence de $s_{n}(\omega)$ vers $s(\omega)$ avec

$$
\int\|s(\omega)\|^{\varepsilon^{\prime}} d \pi(\omega)<+\infty
$$

De plus $s(\omega)-s_{n}(\omega)$ est le reste de la série qui définit $s(\omega)$ et la majoration géométrique précédente fournit aussi une majoration géométrique de l'intégrale de $\left\|s(\omega)-s_{n}(\omega)\right\|^{r^{\prime}}$.

Le Théorème 5 devient ici, avec un énoncé légèrement précisé par rapport à [10] où l'on trouvera en fait la démonstration (Proposition 2 et Théorème 3, §2.2).

ThÉORÈme $5^{\prime}$. Soit $\Gamma$ un sous-groupe de $S l(d, \mathbb{R})$ opérant de manière totalement irréductible et proximale sur $P^{d-1}$. Soit $\mu$ une mesure de probabilité portée par $\Gamma$ telle que $T_{\mu}=\Gamma$ et $\int\|g\|^{c} d \mu(g)<+\infty$ pour un certain c. Soient $X_{n}$ des variables aléatoires indépendantes de loi $\mu, S_{n}$ le produit $X_{n} \cdots X_{1}$, et décomposons $S_{n}$ sous la forme d'Iwasawa $S_{n}=K_{n} A_{n} N_{n}$ avec en particulier $A_{n}=\operatorname{diag}\left(a_{n}^{1}, a_{n}^{2}, \ldots, a_{n}^{d}\right)$. Alors on $a$ pour tout $\varepsilon$ assez petit positif et des constantes $\gamma>0, c>0$ :

$$
\begin{aligned}
& \varlimsup_{n}\left[\int\left|a_{n}^{i+1} / a_{n}^{i}\right|^{*} d \pi(\omega)\right]^{1 / n} \leq 1+C \varepsilon^{2} \quad(i \neq 1, d-1) \\
& \varlimsup_{n}\left[\int\left|a_{n}^{j+1} / a_{n}^{j}\right|^{*} d \pi(\omega)\right]^{1 / n} \leq 1-\gamma \varepsilon \quad(j=1, d-1) .
\end{aligned}
$$

On peut alors énoncer le Théorème $6^{\prime}$ qui, à cause de la présence d'exposants égaux, présente une difficulté supplémentaire par rapport au Théorème 6 . 
ThÉORÈMe 6'. Avec les notations du Théorème $5^{\prime}$, écrivons $S_{n}^{-1}$ sous la forme d'Iwasawa:

$$
S_{n}^{-1}=\eta_{n} \alpha_{n} k_{n} .
$$

Alors la composante $s_{n}$ de $\eta_{n}$ sur V converge p.p. vers set l'on a, pour un certain $\varepsilon>0$ :

$$
\int\left\|s(\omega)-s_{n}(\omega)\right\|^{\varepsilon} d \pi(\omega)<+\infty, \varlimsup_{n}\left[\int\left\|s(\omega)-s_{n}(\omega)\right\|^{\varepsilon} d \pi(\omega)\right]^{1 / n}<1
$$

Preuve. Reprenons la preuve du Théorème 6 en écrivant:

$$
\eta_{n}=\beta_{1} \cdot \alpha_{1}\left(\beta_{2}\right) \cdots \alpha_{n-1}\left(\beta_{n}\right)
$$

avec ici

$$
\beta_{i}=v_{i}^{\prime} m_{i}^{\prime} \in V, m_{i}^{\prime} \in M
$$

et donc

$$
\alpha_{i}\left(\beta_{i}\right)=\alpha_{i}\left(v_{i}^{\prime}\right) \alpha_{i}\left(m_{i}^{\prime}\right)=v_{i} m_{i}
$$

L'action de $\alpha$ sur le vecteur $v^{\prime}$ est celle d'une matrice diagonale de coefficients $\alpha^{k} / \alpha^{d}(k<d)$; il en est de même sur la matrice $m_{i}^{\prime}$ décomposée dans la base canonique et les coefficients sont maintenant $\alpha^{k} / \alpha^{P}(k<P<d)$. On a donc, par application de l'inégalité de Schwarz et du Théorème 5' où $\alpha_{n}=a_{n}^{-1}$, pour $\varepsilon$ assez petit et avec des nouvelles constantes $c>0, \gamma>0$ :

$$
\begin{gathered}
\varlimsup_{n}\left[\int\left\|v_{n}(\omega)\right\|^{F} d \pi(\omega)\right]^{1 / n} \leq 1-\gamma \varepsilon \\
\varlimsup_{n}\left[\int\left\|m_{n}(\omega)\right\|^{F} d \pi(\omega)\right]^{1 / n} \leq 1+C \varepsilon^{2} .
\end{gathered}
$$

Les hypothèses du lemme sont satisfaites pour $\varepsilon$ assez petit et on peut conclure à la convergence de la composante de $\eta_{n}$ sur $V$ vers $s$ avec $\int\|s(\omega)\|^{\varepsilon^{\prime}} d \pi(\omega)<+\infty$ pour un certain $\varepsilon^{\prime}>0$, de même qu'à la majoration géométrique de l'intégrale de $\left\|s(\omega)-s_{n}(\omega)\right\|^{F^{\prime}}$.

Preuve du Théorème 7'. On procède comme au Théorème 7 et on adopte les mêmes notations $g_{i}=x_{i}^{-1}$ et $\nu^{\prime}$ étant la loi limite de $g_{1} \ldots g_{n} \cdot m=\eta_{n} \alpha_{n} . m$. On note de la même façon, les vecteurs $e_{i}$ et les points correspondants. La propriété $\lim _{n} \alpha_{n}^{j} / \alpha_{n}^{d}=0$ $(j<d)$ donne encore ici $\lim _{n} \alpha_{n} \cdot m=\delta_{e_{d}}$ et comme $M$ fixe $e_{d}$ on $a$, la composante de $\eta_{n}$ sur $V$ convergeant sur $s(\omega)$ :

$$
\lim _{n} g_{1} \cdots g_{n} \cdot m=s(\omega) \cdot \delta_{e_{d}}
$$

avec

$$
\int\left\|s(\omega) e_{d}\right\|^{r} d \pi(\omega)<+\infty
$$

On introduit encore $w \in K$ avec $w \cdot e_{d}=e_{1}, k \in K$ avec $k \cdot e_{d}=s \cdot e_{d}, k$ étant défini modulo le statiblisateur $K_{0}$ de $e_{d}$. Soit $\delta$ la métrique quotient sur $P^{d-1}=K / K_{0}$ définie à partir de la norme:

$$
\delta\left(k \cdot e_{d}, k^{\prime} \cdot e_{d}\right)=\operatorname{Inf}_{r \in K_{0}}\left\|k^{-1} k^{\prime}-r\right\|
$$

et comparons $\left\|s e_{d}\right\|$ et $\delta\left(k \cdot e_{d}, e_{1}\right)$ : 
on a encore

$$
\pm k e_{d}\left\|s e_{d}\right\|=s e_{d}
$$

et donc

$$
\left\|s e_{d}\right\|\left|\left\langle k e_{d}, e_{d}\right\rangle\right| \geqslant 1
$$

D'où comme au Théorème 6:

$$
\|k-w\|\left\|s e_{d}\right\| \geq 1
$$

et

$$
\delta\left(k \cdot e_{d}\right)\left\|s e_{d}\right\| \geq 1 .
$$

La condition $\int\|s(\omega)\|^{\varepsilon} d \pi(\omega)$ du Théorème $6^{\prime}$ donne alors

$$
\int\left(1 / \delta^{\varepsilon}\left(\bar{b}, \bar{e}_{1}\right)\right) d \nu^{\prime}(\bar{b})<+\infty
$$

comme au Théorème 6 on conclut grâce aux uniformités valables pour les mesures $\delta_{\sigma} * \mu * \delta_{\sigma-1}(\sigma \in K)$ :

$$
\operatorname{Sup}_{\bar{a} \in P^{d-1}} \int\left(1 / \delta(\bar{a}, \bar{b})^{\varepsilon} d \nu^{\prime}(\bar{b})<+\infty .\right.
$$

Reprenant le calcul précédent, on obtient aussi

$$
\left|\left\langle k e_{d}, e_{d}\right\rangle\right|^{-\varepsilon} \leq\left\|s e_{d}\right\|^{\varepsilon}
$$

et la condition du théorème $6^{\prime}$ donne encore:

$$
\int\left|\left\langle e_{d}, \bar{b}\right\rangle\right|^{-\varepsilon} d \nu^{\prime}(\bar{b})<+\infty
$$

On conclut grâce aux uniformités valables pour les mesures

$$
\begin{gathered}
\delta_{\sigma r} * \mu * \delta_{\sigma-1}(\sigma \in K) \\
\operatorname{Sup}_{a \in P^{\prime-1}} \int|\langle a, b\rangle|^{-\varepsilon} d \nu^{\prime}(b)<+\infty
\end{gathered}
$$

2.9. Spectre d'un produit de matrices aleatoires

L'objet de ce paragraphe est de prouver le.

ThÉorème 8. Soit $\mu$ une mesure de probabilité sur $\mathrm{Sl}(d, \mathbb{R})$ telle que $T_{\mu}$ agisse de façon proximale et totalement irréductible sur $P^{d-1}$. On suppose qu'il existe $c>0$ avec $\int\|g\|^{c} d \mu(g)<+\infty$. Alors, presque sûrement, le produit de matrices aléatoires $S_{n}(\omega)$ admet, pour tout $n$ assez grand, une valeur propre dominante réelle simple $\lambda_{n}$. On a aussi, avec $\gamma=\lim n^{-1} \log \left\|S_{n}\right\|$

$$
\text { p.p. } \quad \lim \frac{1}{n} \log \left|\lambda_{n}\right|=\gamma .
$$

Ce théorème découlera du suivant.

THÉORĖME 9. Avec les notations et hypothèses du Théorème 8, la trace de $S_{n}(\omega)$ vérifie: p.p. $\lim n^{-1} \log \left|\operatorname{Tr} S_{n}\right|=\gamma$. 
Ce théorème découle de plusieurs lemmes.

Lemme 7. Ecrivons $S_{n}$ dans la décomposition d'Iwasawa $S_{n}=K_{n} A_{n} N_{n}$. Alors $\operatorname{Tr}\left(S_{n}\right)=$ $\sum_{i=1}^{d} a_{n}^{i}\left\langle K_{n} e_{i}, N_{n}^{\prime} e_{i}\right\rangle$.

Preuve. Il suffit de noter que $\operatorname{Tr} S_{n}=\operatorname{Tr}\left(N_{n} K_{n} A_{n}\right)$ et

$$
\left\langle N_{n} K_{n} A_{n} e_{i}, e_{i}\right\rangle=a_{n}^{i}\left\langle K_{n} e_{i}, N_{n}^{\prime} e_{i}\right\rangle .
$$

Dans la suite on doit étudier la matrice triangulaire inférieure $N_{n}^{t}$ qui apparaît dans l'écriture $S_{n}^{\prime}=N_{n}^{\prime} A_{n} K_{n}^{-1}$. L'étude de $S_{n}^{\prime}=X_{1}^{\prime} X_{2}^{\prime} \cdots X_{n}^{\prime}=g_{1}^{\prime} g_{2}^{\prime} \cdots g_{n}^{\prime}$ est analogue à celle de $S_{n}^{-1}=g_{1} g_{2} \cdots g_{n}$ puisque les hypothèses de proximalité et d'irréductibilité valent aussi bien pour $T_{\mu}^{\prime}$ que pour $T_{\mu}$ ou $T_{\mu}^{-1}$; il y a en particulier convergence de $N_{n}^{\prime} e_{1}$ vers $t(\omega)$ à vitesse exponentielle: $\overline{\lim }\left[\int\left\|N_{n}^{t} e_{1}-t(\omega)\right\|^{*} d \pi(\omega)\right]^{1 / n}<1$ pour un $\varepsilon>0$ convenable.

On a vu également en [10] que la projection $S_{n} \cdot e_{1}$ de $S_{n}$ sur $P^{d-1}$, qui correspond au vecteur unitaire $K_{n} e_{1}$, converge en loi vers la mesure invariante $\nu$ et que cette convergence a lieu à vitesse exponentielle au sens que l'on précise ci-dessous. Si $\varphi$ est une fonction Holdérienne d'ordre $\varepsilon$ sur un espace métrique $(X, \delta)$ on note

$$
[\varphi]_{F}=\operatorname{Sup}_{x, y \in X} \frac{|\varphi(x)-\varphi(y)|}{\delta^{F}(x, y)} .
$$

On a alors, pour $\varepsilon$ assez petit, l'existence de deux constantes $C$ et $\rho \in] 0,1[$ avec, pour toute $\varphi \varepsilon$-Holdérienne sur $P^{d-1}$ :

$$
\operatorname{Sup}_{x \in P^{d-1}}\left|\int \varphi\left(S_{n} \cdot x\right) d \pi(\omega)-\nu(\varphi)\right| \leq C \rho^{n}[\varphi]_{\digamma} .
$$

Lemme 8. Considérons le couple $S_{n} \cdot e_{1}, N_{n}^{\prime} e_{1}$ de $P^{d-1} \times \mathbb{R}^{d-1}$. Il existe $\varepsilon>0, C>0$ et $\rho \in\left[0,1\left[\right.\right.$ tels que pour toute fonction $\varepsilon$-Holdérienne sur $P^{d-1} \times \mathbb{R}^{d-1}$ on ait:

$$
\left|\int \varphi\left(S_{n} \cdot e_{1}, N_{n}^{\prime} e_{1}\right) d \pi(\omega)-\int \varphi[x, t(\omega)] d \nu(x) d \pi(\omega)\right| \leq C \rho^{n}[\varphi]_{\varepsilon} .
$$

Preuve. La différence précédente est somme des 3 termes $\Delta_{1}, \Delta_{2}, \Delta_{3}$ suivants:

$$
\begin{aligned}
& \Delta_{1}=\int \varphi\left(S_{n} \cdot e_{1}, N_{n}^{\prime} e_{1}\right) d \pi(\omega)-\int \varphi\left(S_{n} \cdot e_{1}, N_{[n / 2]}^{\prime} e_{1}\right) d \pi(\omega) \\
& \Delta_{2}=\int \varphi\left(S_{n} \cdot e_{1}, N_{[n / 2]}^{\prime} e_{1}\right) d \pi(\omega)-\int \varphi\left(x, N_{[n / 2]}^{\prime} e_{1}\right) d \nu(x) d \pi(\omega) \\
& \Delta_{3}=\int \varphi\left(x, N_{[n / 2]}^{\prime} e_{1}\right) d \nu(x) d \pi(\omega)-\int \varphi[x, t(\omega)] d \nu(x) d \pi(\omega) .
\end{aligned}
$$

Les remarques précédant le lemme donnent les majorations, avec un certain $\rho \in] 0,1[$ et une constante $C>0$

$$
\begin{aligned}
& \left|\Delta_{1}\right| \leq[\varphi]_{F}\left[\int\left\|N_{n}^{\prime} e_{1}-t(\omega)\right\|^{*} d \pi(\omega)+\int\left\|N_{[n / 2]}^{\prime} e_{1}-t(\omega)\right\|^{*} d \pi(\omega)\right] \\
& \left|\Delta_{1}\right| \leq C[\varphi]_{F} \rho^{[n / 2]}+c[\varphi]_{F} \rho^{n} \\
& \left|\Delta_{2}\right| \leq C[\varphi]_{F} \rho^{[n / 2]} \\
& \left|\Delta_{3}\right| \leq C[\varphi]_{F} \rho^{[n / 2]} .
\end{aligned}
$$


On a donc l'inégalité voulue en modifiant $C$ et $\rho$. Dans la majoration de $\Delta_{2}$ on a utilisé l'indépendance des $X_{i}$ en fixant d'abord [n/2] variables et en intégrant par rapport aux variables $X_{i}$ pour $i>[n / 2]$.

Lemme 9. Notons y l'image canonique de $y \in N^{\prime} e_{1}$ dans $P^{d-1}$. Alors la fonction sur $P^{d-1} \times \mathbb{R}^{d-1}$ définie par $u(x, y)=|\langle x, \bar{y}\rangle|$ est Lipchitzienne.

Preuve. $|\langle x, \bar{y}\rangle|$ est bien défini en considérant $x$ et $\bar{y}$ comme des vecteurs unitaires définis au signe près. Alors:

$$
|\langle x, \bar{y}\rangle|-\left|\left\langle x^{\prime}, \bar{y}^{\prime}\right\rangle\right| \leq\left\|x-x^{\prime}\right\|+\left\|\bar{y}-\bar{y}^{\prime}\right\| .
$$

En modifiant les vecteurs par le changement désigné:

$$
\left|u(x, \bar{y})-u\left(x^{\prime}, \bar{y}^{\prime}\right)\right| \leq \delta\left(x, x^{\prime}\right)+\delta\left(\bar{y}, \bar{y}^{\prime}\right) .
$$

Pour $\beta>0$, notons $\psi_{\beta}$ la fonction linéaire par morceaux égale à 1 sur $\left[-e^{-\beta}, e^{\beta}\right]$, nulles en dehors de $2\left[-e^{-\beta}, e^{-\beta}\right]$.

LEMME 10. Pour tout $\alpha>0$ on a

$$
\sum_{n=1}^{\infty} \int \psi_{n \alpha} \circ u[x, t(\omega)] d \nu(x) d \pi(\omega)<\infty
$$

Preuve. Utilisons le Théorème 7':

$$
\operatorname{Sup}_{y \in P^{i-1}} \int|\langle x, y\rangle|^{-\varepsilon} d \nu(x)=C<+\infty
$$

avec $y$ égal au point correspondant à $t(\omega)$ dans $P^{d-1}$. On obtient

$$
\nu\left\{x ;|\langle x, y\rangle| \leq 2 e^{-n \alpha}\right\} \leq C e^{-n a \varepsilon_{2} \varepsilon}
$$

et

$$
\int \psi_{n \alpha x} \circ u[x, t(\omega)] d \nu(x) d \pi(\omega) \leq C 2^{\varepsilon} e^{-n \alpha \varepsilon} .
$$

La série de ces intégrales est donc convergente.

LEMME 11.

$$
\operatorname{Lim}_{n} \frac{1}{n} \log \left|\left\langle K_{n} e_{1}, N_{n}^{\prime} e_{1}\right\rangle\right|=0 .
$$

Preuve. Comme $N_{n}^{t} e_{1}$ converge la quantité $u_{n}=\left|\left\langle K_{n} e_{1}, N_{n}^{\prime} e_{1}\right\rangle\right|$ est bornée et il suffit de voir que, pour tout $\alpha>0$

$$
\pi\left\{\lim \frac{1}{n} \log u_{n}<-\alpha\right\}=0 .
$$

Ceci découlera de

$$
\sum_{n} \pi\left\{u_{n}<e^{-n+x}\right\}<+\infty
$$

c'est-à-dire de

$$
\sum_{n} \int \psi_{n r r}\left(\left\langle K_{n} \cdot e_{1}, N_{n}^{\prime} \cdot e_{1}\right\rangle\right) d \pi(\omega)<+\infty .
$$

Cette finitude découle du Lemme 8 en prenant $\varphi=\psi_{n c r} \circ u$ et du Lemme 10. 
LEMME 12.

$$
\varlimsup_{n}\left|\frac{a_{n}^{i}}{a_{n}^{1}}\left\langle K_{n} e_{i}, N_{n}^{\prime} e_{i}\right\rangle\right|^{1 / n}<1 \quad(i>1) .
$$

Preuve. Utilisons comme au paragraphe précédent la décomposition du groupe $N^{t}$ sous forme d'un produit semi-direct d'un espace vectoriel $V=\mathbb{R}^{d-1}$ et d'un groupe $M$ fixant $e_{1}$. Alors $N_{n}^{\prime}$ s'écrit

$$
N_{n}^{\prime}=v_{n} m_{n} \quad \text { avec } \quad v_{n} e_{i}=e_{i}(i>1)
$$

et $m_{n} e_{1}=e_{1}$; il en découle

$$
\left\|N_{n}^{\prime} e_{i}\right\|=\left\|m_{n} e_{i}\right\|<\left\|m_{n}\right\| \quad(i>1) .
$$

Il en découle

$$
\left|\frac{a_{n}^{i}}{a_{n}^{1}}\left\langle K_{n} e_{i}, N_{n}^{\prime} e_{i}\right\rangle\right| \leq\left|\frac{a_{n}^{i}}{a_{n}^{1}}\right|\left\|N_{n} e_{i}\right\| \leq\left|\frac{a_{n}^{i}}{a_{n}^{i}}\right|\left\|m_{n}\right\| .
$$

Utilisant les relations

$$
\begin{aligned}
& \varlimsup_{n}\left[\int\left\|m_{n}(\omega)\right\|^{\varepsilon} d \pi(\omega)\right]^{1 / n} \leq 1+C \varepsilon^{2} \\
& \varlimsup_{n}\left[\int\left|\frac{a_{n}^{i}}{a_{n}^{i}}(\omega)\right|^{\varepsilon} d \pi(\omega)\right]^{1 / n} \leq 1-\gamma \varepsilon \quad(i>1)
\end{aligned}
$$

en prenant $\varepsilon$ assez petit

$$
\varlimsup_{n}\left[\int\left|\frac{a_{n}^{i}}{a_{n}^{1}}(\omega)\right|^{F / 2}\left\|m_{n}\right\|(\omega)^{F / 2} d \pi(\omega)\right]^{1 / n}<1 .
$$

La série de terme général $\left|a_{n}^{i} / a_{n}^{1}\right|^{F / 2}\left\|m_{n}(\omega)\right\|^{F / 2}$ est donc convergente et son terme général tend vers zéro géométriquement.

Preuve du Théorème 9. Les Lemmes 7, 11 et 12 montrent que $\lim _{n}\left(\left|\operatorname{Tr} S_{n}\right| /\left|a_{n}^{1}\right|\right)^{1 / n}=1$. D'où:

$$
\lim _{n} \frac{1}{n} \log \left|T r S_{n}\right|=\lim _{n} \frac{1}{n} \log \left|a_{n}^{1}\right|=\gamma .
$$

La preuve du Théorème 8 découlera du.

LEMME 13. On considère l'équation de degré $d: \lambda^{d}+\theta_{1} \lambda^{d-1}+\cdots+\theta_{d}=0$ où les $\theta_{i}$ sont réels. Alors pour tout $\varepsilon>0$, il existe $\eta>0$ tel que la condition $\operatorname{Sup}_{1<k<d}\left|\theta_{k} / \theta_{1}^{k}\right| \leq \eta$ implique l'existence $d$ 'une unique racine simple réelle $\lambda_{1}$ de l'équation de module maximum et vérifiant

$$
\left|\frac{\lambda_{1}}{\theta_{1}}+1\right|<\varepsilon
$$

Preuve. Le changement d'inconnue $\lambda=-\theta_{1} x$ ramène à l'équation

$$
x^{d}-x^{d-1}+\alpha_{2} x^{d-2}+\cdots+\alpha_{d}=0
$$

evec

$$
\alpha_{k}=\theta_{k} / \theta_{1}^{k}
$$


Donc lorsque Sup $\operatorname{Su}_{1 \leq k \leq d}\left|\alpha_{k}\right|$ tend vers zéro, cette dernière équation tend vers $x^{d}-$ $x^{d-1}=0$, qui admet +1 pour racine multiple d'ordre $d-1$.

Le théorème des fonctions implicites donne alors l'existence d'une racine $x^{\prime}$ réelle simple proche de 1 pour $\eta$ assez petit, de façon que $\left|x^{1}-1\right|<\varepsilon$. Alors les fonctions symmétriques des autres racines sont arbitrairement petites et il en est donc de même des racines: la racine $x_{1}$ est bien dominante. En revenant à l'équation initiale on obtient le résultat annoncé.

Preuve du Théorème 8. Considérons l'action de $T_{\mu}$ sur les puissances extérieures de $\mathbb{R}^{d}$ et notons $S_{n}^{k}$ la matrice correspondant à $S_{n}$ dans $\Lambda^{k} \mathbb{R}^{d}(1 \leq k \leq d)$. La propriété de proximalité et d'irréductibilité de $T_{\mu}$ implique [12] la simplicité du plus grand exposant de Liapunoff et donc

$$
\lim _{n}\left(\left\|S_{n}^{k}\right\| /\left\|S_{n}\right\|\right)^{1 / n}<1 \quad(k>1)
$$

Les coefficients $\theta_{k}$ du polynome caractéristique de $S_{n}$ satisfont $\left|\theta_{k}\right|=\left|\operatorname{Tr} S_{n}^{k}\right| \leq d\left\|S_{n}^{k}\right\|$.

Comme d'après le Théorème $9 \lim _{n}\left\|S_{n}\right\|^{1 / n}=\lim _{n}\left|\operatorname{Tr} S_{n}\right|^{1 / n}$ on a bien pour $k>1: \theta_{k} / \theta_{1}^{k}$ arbitrairement petit. Le Lemme 12 donne alors l'existence de la valeur propre dominante simple, réelle $\lambda_{n}$ de $S_{n}$ avec de plus

$$
\lim _{n}\left|\lambda_{n}\right| /\left|\operatorname{Tr} S_{n}\right|=1 \text {. }
$$

Le Théorème 9 donne encore

$$
\lim _{n} \frac{1}{n} \log \left|\lambda_{n}\right|=\lim _{n} \frac{1}{n} \log \left|\operatorname{Tr} S_{n}\right|=\gamma
$$

Remarque. La méthode de preuve du Théorème 8 étant basée sur le théorème des fonctions implicites, son énoncé reste valable lorsque $\mathbb{R}$ est remplacé par un corps local: il y a une valeur propre dominante appartenant à ce corps lui-même.

THÉORÈme 8'. Soit $\mu$ une mesure de probabilité sur $\mathrm{Sl}(d, \mathbb{R})$ telle que $T_{\mu}$ opère de façon proximale et totalement irréductible sur la frontière de Fürstenberg $B$. On suppose $q u^{\prime}$ il existe $c>0$ avec $\int\|g\|^{c} d \mu(g)<+\infty$. Alors, presque sûrement, pour $n$ assez grand, le produit de matrices aléatoires $S_{n}(\omega)$ est diagonalisable à valeurs propres réelles distinctes $\lambda_{n}^{k}\left(\left|\lambda_{n}^{1}\right|>\left|\lambda_{n}^{2}\right|>\cdots>\left|\lambda_{n}^{d}\right|\right)$. Si l'on note $\gamma^{k}$ le $k^{\text {ìme }}$ exposant de Liapunoff de $S_{n}\left(\gamma^{\prime}=\gamma\right)$ on a presque sûrement:

$$
\lim _{n} \log \left|\lambda_{n}^{k}\right|=\gamma^{k}
$$

Preuve. L'hypothèse sur $T_{\mu}$ assure la simplicité du spectre de Liapunoff [10]. On peut appliquer le Théorème 8 aux différentes puissances extérieures de $\mathbb{R}^{d}$ et on a donc l'existence d'une valeur propre dominante réelle dans un tel produit avec

$$
\lim _{n} \frac{1}{n} \log \left|\lambda_{n}^{1} \ldots \lambda_{n}^{k}\right|=\gamma^{1}+\cdots \gamma^{k}
$$

Donc, pour tout $k \leq d: \lim _{n} n^{-1} \log \left|\lambda_{n}^{k}\right|=\gamma^{k}$ et la conclusion voulue.

Remarque. Comme le Théorème 8 , ce théorème reste valable sur un corps local. 


\subsection{Remarques et questions}

(1) Les résultats de la fin de la partie 2 peuvent être précisés: la valeur propre dominante $\lambda_{n}(\omega)$ a, en module, un comportement très proche de celui de $S_{n}(\omega)$ : elle suit les mêmes lois limites du calcul des probabilités [10]. De plus le point attractif de $S_{n}(\omega)$ converge en loi tandis que le point répulsif converge p.p., avec indépendance asymptotique de ces variables aléatoires. On peut conjecturer que, pour presque tout $\left(\omega, \omega^{\prime}\right) \in \Omega \times \Omega$, et $n$ assez grand, $S_{n}(\omega)$ et $S_{n}\left(\omega^{\prime}\right)$ engendrent un groupe libre.

(2) Il serait utile de préciser dans quelles conditions la mesure harmonique $\nu$ est absolument continue par rapport à la mesure de Lebesgue ainsi que de préciser les harmoniques bornées associées [16].

(3) Quelles relations y a-t-il entre l'ensemble des points limites $L_{\mathrm{l}}$ et l'ensemble des points non errants sous l'action de $\Gamma$. Ces notions devraient peut-être ici être adaptées.

(4) L'ensemble $L_{1}$ peut-il être de mesure de Lebesgue positive sans être égal à $2[22]$ ?

(5) Les techniques précédentes permettent-elles de discuter l'existence de sousgroupes libres dans les groupes non moyennables d'homéomorphismes de variétés compactes?

(6) Existe-t-il des mesures $\nu$ portées par $L_{\mathrm{l}}$ qui soient $\mu$-invariantes pour un $\mu$ convenable et de 'dimension' égale à celle de $L_{\mathrm{r}}$ ?

\section{Acknowledgements}

L'auteur remercie P. de la Harpe pour d'utiles commentaires concernant le théorème de Tits et les propriétés des algèbres de groupe. Il remercie également $\mathrm{H}$. Fürstenberg pour une preuve simplifiée de la positivité de la dimension de Hausdorff de $L_{1}$.

\section{REFERENCES}

[1] P. Bougerol \& J. Lacroix. Products of Random Matrices with Applications to Schrödinger Operators. Birkhauser: 1986.

[2] C. Chevalley. Théorie des Groupes de Lie. Hermann: 1968.

[3] J. Cohen, H. Kesten \& C. Newman, eds., Random matrices and applications. Contemp. Math. Amer. Math. Soc. 50 (1986).

[4] J. D. Dixon. Free subgroups of linear groups. pp. 45-56. Lecture Notes in Math. 319 Springer: 1973.

[5] H. Furstenberg. Boundary theory and stochastic processes on homogeneous spaces. Proc. Symp. Pure Math. 6 (1972), 193-229.

[6] S. Glaner. Proximal flows. Springer Lecture Notes 517 (1976).

[7] 1. Goldsheid \& G. A. Margulis. Simplicity of the Liapunoff spectrum for products of random matrices. 35 (1987), 309-313.

[8] F. Guimier. Simplicité du spectre de Liapunoff d'un produit de matrices aléatoires sur un corps ultramétrique. C.R.A.S. Paris (1989), (à paraître).

[9] Y. Guivarc'h. Quelques propriétés asymptotiques des produits de matrices aléatoires. Springer Lecture Notes 774 (1980) 176-250.

[10] Y. Guivarc'h \& A. Raugi. Frontière de Furstenberg, propriétés de contraction et théorèmes de convergence. Z. Wahr. 69 (1985) 187-242.

[11] Y. Guivarc'h. Croissance polynomiale et périodes des fonctions harmoniques. Bulletin Soc. Marh. France 101 (1973), 333-379. 
[12] Y. Guivarc'h \& A. Raugi. Propriétés de contraction d'un semi-groupe de matrices inversibles. Israël J. Math. 65 (2) (1989), 165-196.

[13] Y. Guivarc'h \& A. Raugi. Quelques remarques sur les produits de matrices aléatoires indépendantes. C.R.A.S. 304 série 1, 8 (1987), 199-201.

[14] P. de la Harpe. Free groups in linear groups. L'Enseignement Math. 29 (1983), 129-144.

[15] P. de la Harpe. Reduced $C^{*}$-algebras of discrete groups which are simple with a unique trace. pp. 248-251. Lecture Notes 1132 Springer-Verlag.

[16] F. Ledrappier. Poisson boundaries of discrete groups of matrices. Israël J. Math. 50 (4) (1985).

[17] E. Le Page. Théorèmes limites pour les produits de matrices aléatoires. Springer Lecture Notes 928 (1982), 258-303.

[18] G. A. Margulis. Arithméticity of the irreductible lattices in the semi-simple groups of rank greater than one. Invent. Math. 76 (1984), 93-120.

[19] G. A. Margulis \& G. A. Soifer. A criterion for the existence of maximal subgroups of infinite index in a finitely generated linear group. Soviet Math. Dokl. 18 (3) (1977), 847-851.

[20] C. C. Moore. Amenable subgroups of semi-simple groups and proximal flows. Israël J. Math. 34 (1979), 121-138.

[21] M. S. Raghunathan. A proof of Oseledet multiplicative theorem. Israël J. Math. 32 (1979) 356-362.

[22] D. Sullivan. The density at infinity of a discrete group of hyperbolic motions. Publ. Math. I.H.E.S. 50.

[23] J. Tits. Free subgroups in linear groups. J. of Algebra 20 (1972), 250-270.

[24] B. A. F. Wehrfritz. Infinite linear groups. Ergebnisse der Marhematik (1973).

[25] A. Weil. Basic number theory. Grundlehren der Math Wiss. 144 Springer: 1967.

[26] R. Zimmer. Ergodic theory and semi-simple groups. Birkhauser: 1984. 